\title{
TU/e EmonONEN

\section{Simulation techniques for spatially evolving instabilities in compressible flow over a flat plate}

\section{Citation for published version (APA):}

Wasistho, B., Geurts, B. J., \& Kuerten, J. G. M. (1997). Simulation techniques for spatially evolving instabilities in compressible flow over a flat plate. Computers \& Fluids, 26(7), 713-739. https://doi.org/10.1016/S00457930(97)00021-2

DOI:

10.1016/S0045-7930(97)00021-2

Document status and date:

Published: 01/01/1997

\section{Document Version:}

Publisher's PDF, also known as Version of Record (includes final page, issue and volume numbers)

\section{Please check the document version of this publication:}

- A submitted manuscript is the version of the article upon submission and before peer-review. There can be important differences between the submitted version and the official published version of record. People interested in the research are advised to contact the author for the final version of the publication, or visit the $\mathrm{DOI}$ to the publisher's website.

- The final author version and the galley proof are versions of the publication after peer review.

- The final published version features the final layout of the paper including the volume, issue and page numbers.

Link to publication

\section{General rights}

Copyright and moral rights for the publications made accessible in the public portal are retained by the authors and/or other copyright owners and it is a condition of accessing publications that users recognise and abide by the legal requirements associated with these rights.

- Users may download and print one copy of any publication from the public portal for the purpose of private study or research.

- You may not further distribute the material or use it for any profit-making activity or commercial gain

- You may freely distribute the URL identifying the publication in the public portal.

If the publication is distributed under the terms of Article 25fa of the Dutch Copyright Act, indicated by the "Taverne" license above, please follow below link for the End User Agreement:

www.tue.nl/taverne

Take down policy

If you believe that this document breaches copyright please contact us at:

openaccess@tue.nl

providing details and we will investigate your claim. 


\title{
SIMULATION TECHNIQUES FOR SPATIALLY EVOLVING INSTABILITIES IN COMPRESSIBLE FLOW OVER A FLAT PLATE
}

\author{
B. WASISTHO, B. J. GEURTS and J. G. M. KUERTEN \\ Department of Applied Mathematics, J. M. Burgers Centre, University of Twente, P.O. Box 217, \\ 7500 AE, Enschede, The Netherlands
}

(Received 25 March 1996; in revised form 31 March 1997)

\begin{abstract}
In this paper we present numerical techniques suitable for a direct numerical simulation in the spatial setting. We demonstrate the application to the simulation of compressible flat plate flow instabilities. We compare second and fourth order accurate spatial discretization schemes in combination with explicit multistage time stepping for the simulation of the 2D Navier-Stokes equations. We consider Mach numbers 0.5 and 4.5. In the vicinity of the outflow boundary, an efficient buffer domain treatment is introduced, which is suitable in conjunction with an explicit time integration scheme. This treatment requires only a short buffer domain to damp wave reflections at the outflow boundary. Results for the instability of Tollmien-Schlichting ( $T-S)$ waves are compared with two instability theories, linear stability theory (LST) and linear parabolized stability equations (PSE). The growth rates of T-S waves for parallel base flow at both Mach numbers compare well with LST results. Moreover, the growth rates of T-S waves for nonparallel base flow compare well with results obtained by solving the PSE at Mach number 0.5 . The second order discretization scheme requires, however, considerably higher grid resolution than the fourth order method to achieve accurate results. High amplitude disturbances were also considered to activate nonlinear terms. The nonlinearity strongly affects the form of the $T-S$ waves and the growth rate of the disturbances. The results obtained here support the use of these numerical techniques in flow simulations with increasing complexity such as flat plate flow simulations up to the turbulent regime and with separation regions in $3 \mathrm{D}$. The results also encourage the use of perturbations derived from the compressible PSE as inlet perturbations for nonparallel flow. (C) 1997 Elsevier Science Ltd
\end{abstract}

\section{INTRODUCTION}

Direct numerical simulation of the Navier-Stokes equations has been the subject of many intensive studies. The numerical simulations of spatially evolving flows are constrained by artificial boundary conditions, which need to be introduced in order to limit the computational domain. In this paper we focus on numerical simulations of viscous compressible flow over a flat plate. In the literature, most simulations of this flow are performed using periodic boundary conditions in the streamwise direction, and the computational frame is moving with a 'characteristic' velocity, the phase velocity, along the plate [1]. The advantage of this so-called 'temporal setting' is that only a relatively small computational domain is needed in the streamwise direction and very accurate numerical methods such as spectral or pseudo-spectral spatial discretization can be used in the downstream direction $[1,2]$. The periodicity assumption, however, limits the applicability of these simulations and renders a direct comparison with physical experiment difficult. In a more general setting there is no periodicity in the streamwise direction and artificial inflow and outflow boundaries are needed. In this configuration, the computational frame is fixed in space and the flow is entering the domain through the inflow boundary and leaving it through the outflow boundary, i.e. the simulations are performed in a so-called 'spatial setting'. The major drawback of this type of simulation is that a much larger streamwise extent of the computational domain is required and hence the computational effort is considerably increased. The main difficulty of these spatial simulations lies in providing consistent and accurate inflow and outflow boundary conditions. The boundary conditions should be consistent in order to ensure the well-posedness of the Navier-Stokes equations. Moreover, a proper inflow boundary condition should let the flow enter the computational domain such that a minimum downstream region is needed for the flow to adjust itself to the solution within the domain. On the other hand, an outflow boundary condition is 
needed which prevents spurious numerical reflections which can disturb the solution within the domain as much as possible. Stable outflow boundary conditions for compressible viscous flow are difficult to specify, especially when large disturbances are considered. The use of an additional buffer domain in the vicinity of the outflow boundary is indispensable, in particular for subsonic flow, in order to efficiently damp wave reflections from the outflow boundary.

In this paper, the spatially evolving boundary layer over a flat plate is studied in $2 \mathrm{D}$. The computational domain has the form of a rectangle as shown in Fig. 1. The domain is bounded by a solid wall at the lower side, while the other sides are open boundaries treated as artificial boundaries. This configuration provides a computationally affordable framework for the development of suitable numerical techniques for the simulation of a transition process from laminar to turbulent flow. Wc focus on two aspccts, i.e. the spatial discretization method and the treatment of the artificial boundaries, primarily the outflow boundary. Highly accurate spatial discretization is necessary in order to be able to favourably compare a DNS result with solutions of simplified mathematical models, such as the linear stability theory (LST) and parabolized stability equations (PSE). The best suited methods for this purpose are spectral [3] and high order central difference approximations. In this paper, a fourth order accurate central scheme is developed which is favoured in view of an extension to simulations employing non-orthogonal grids in the future.

Regarding the artificial boundaries, various techniques for treating these boundaries for compressible viscous flows have been proposed in the literature, among others by Rudi-Strikwerda [4], Thompson [5], and Poinsot-Lele [6]. The number of boundary conditions needed for the well-posedness of the Navier-Stokes equations has been mathematically proven by Strikwerda [7]. The proposed methods are generally based on characteristic wave relations derived from inviscid fluid dynamics equations. An alternative for defining these boundary conditions is based on an extrapolation method [4]. Our experiences with the various boundary treatments resulted in some preferences regarding the choice of the boundary treatment in the case where flow over a flat plate is concerned [8]. For the inflow boundary, an extrapolation method appears prefcrable whereas 'non-reflecting' boundary conditions based on characteristic wave methods appear more suitable at the freestream and the outflow boundary. For time-dependent problems, the introduction of an additional buffer domain can strongly enhance the quality of the solutions, in particular for subsonic flow. The focus in this work is on the improvement of the outflow boundary treatment by adding a buffer domain in the vicinity of the outflow boundary, in which the disturbances are strongly reduced and the flow is gradually brought back to the laminar base flow. Various approaches are presented in the literature, for example increasing viscosity [9], increasing grid spacing [10], acceleration of the base flow [11], parabolization of the governing equations $[9,11,12]$ and gradually suppressing the disturbances $[12,13]$. Our experiments with these approaches shows that increasing viscosity and employing a damping function yield more robust methods than the other two techniques. The approach of strongly increasing viscosity is, however, less efficient in suppressing the disturbances than multiplying the disturbances directly

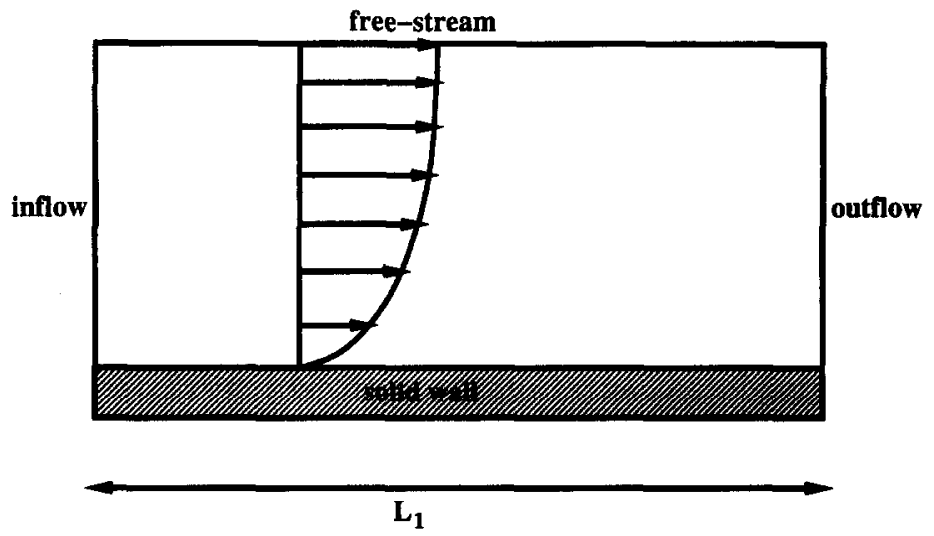

Fig. 1. Configuration of the computational domain bounded by a solid wall and open 'artificial' boundaries. 
by a damping function in the buffer domain. Moreover, the former approach has an important disadvantage when an explicit time stepping method is used, since it decreases the allowable time step considerably. Based on this experience, a buffer domain treatment using an efficient damping function is introduced in this paper. This buffer domain treatment is extensively validated and formulated such that it is insensitive to the flow configuration and the grid density.

The simulations are performed at Mach number 0.5 and 4.5 . In the supersonic flow we consider parallel base flow with small disturbances, while in the subsonic flow we study both parallel and nonparallel base flows with small as well as large disturbances. This diverse set of flow conditions results in suitable test cases with which the performance of the discretization schemes and the artificial boundary treatments can be examined systematically. This set of test cases corresponds to simulations which are successively more critical to the performance of the developed techniques. A subsonic flow simulation for instance is more critical for the performance of the outflow boundary treatments than a supersonic flow due to larger upstream influences. Furthermore, large disturbances form a more difficult test for the outflow treatments and the discretization scheme. Specifically, for the validation the parallel flow results are directly compared with LST predictions. On the other hand, results of nonparallel flow simulations are compared with the solution of linear PSE. In particular, we pay attention to a correct prediction of the growth rates, which forms a very critical test. Nonlinear effects in the latter flow become appreciable when we introduce large disturbances at the inflow boundary.

The paper is organized as follows. In Section 2 we outline the governing equations, while the numerical method is described in Section 3. Section 4 gives a description of the artificial boundary conditions and the buffer domain treatment. Section 5 provides a discussion of simulation results, and finally we summarize our findings in Section 6.

\section{GOVERNING EQUATIONS}

Compressible flow over a flat plate can be described by the time-dependent Navier-Stokes equations which represent conservation of mass, momentum and energy:

$$
\begin{gathered}
\partial_{t} \rho+\partial_{j}\left(\rho u_{j}\right)=0 \\
\partial_{,}\left(\rho u_{i}\right)+\partial_{j}\left(\rho u_{i} u_{j}\right)+\partial_{i} p-\partial_{j} \tau_{i j}=0 \\
\partial_{,} e+\partial_{j}\left((e+p) u_{j}\right)-\partial_{j}\left(\tau_{i j} u_{i}-q_{j}\right)=0 .
\end{gathered}
$$

The symbols $\partial_{t}$ and $\partial_{j}$ denote the partial differential operators $\partial / \partial_{t}$ and $\partial / \partial x_{j}$ with respect to time $(t)$ and spatial coordinate $\left(x_{j}\right)$, respectively; $\rho$ is the density, $p$ the pressure, $u_{i}$ the $i$ th component of the velocity vector, and $e$ the total energy density, given by

$$
e=\frac{p}{\gamma-1}+\frac{1}{2} \rho u_{i} u_{i}
$$

where $\gamma$ denotes the adiabatic gas constant. Moreover, $\tau_{i j}$ is the stress tensor which is a function of the dynamic viscosity $\mu$ and velocity vector $u$ :

$$
\tau_{i j}=\frac{\mu(T)}{\operatorname{Re}}\left(\partial_{i} u_{i}+\partial_{i} u_{i}-\frac{2}{3} \delta_{i j} \partial_{k} u_{k}\right)
$$

where $\operatorname{Re}=\left(\rho_{x} u_{c c} \delta^{*}\right) / \mu\left(T_{x}\right)$ is the reference Reynolds number. The dynamic viscosity $\mu$ can be constant or related to the temperature $T$ by Sutherland's law. Finally, $q_{j}$ is the viscous heat flux vector, defined as

$$
q_{j}=-\frac{\mu}{(\gamma-1) \operatorname{RePr} M_{x}^{2}} \partial_{j} T
$$


where $P r$ is the Prandtl number and $M_{x}$ is the reference Mach number. The temperature $T$ is related to the density $\rho$ and the pressure $p$ by the ideal gas law

$$
T=\gamma M_{\times}^{2} \frac{p}{\rho} .
$$

Throughout we use $\gamma=1.4$ and $\operatorname{Pr}=0.72$. The values of the reference Mach number $M_{x}$ and the Reynolds number $R e$ are specified for each case separately. The above variables have been made dimensionless using reference scales, i.e. a reference length $\delta^{*}$, which is the boundary layer displacement thickness, density $\rho_{x}$, velocity $u_{x}$, temperature $T_{x}$ and viscosity $\mu\left(T_{x}\right)$. The subscript $\infty$ refers to the freestream value.

$\Lambda$ no-slip isothermal boundary condition is imposed at the wall. Specifically, the velocity components vanish, the wall temperature is prescribed and the pressure is extrapolated from the interior points consistent with the auxiliary condition $\partial_{2} p=0$. Hence, no conservation equation needs to be solved along the wall. The inflow, the outflow and the freestream boundary treatments are discussed in detail in Section 4.

In the parallel flow simulations reported in Section 5, the initial base flow is formed by the compressible Blasius boundary layer which is extended such that it is invariant in the streamwise direction and in which the normal velocity is set to zero. In the nonparallel flow simulations, the initial base flow is the full similarity solution to the compressible boundary layer equations. This implies that, given the compressible Blasius solution at some location $x_{1}$, the solution at a different location $\hat{x}_{1}$ can be found straightforwardly provided we scale $\hat{x}_{2}=\sqrt{\hat{x}_{1} / x_{1} x_{2}}$. Specifically, if $\zeta$ denotes any component of the solution, then $\zeta\left(\hat{x}_{1},\left(\hat{x}_{1} / x_{1}\right)^{1 / 2} x_{2}\right)=\zeta\left(x_{1}, x_{2}\right)$. In this case, the normal velocity is nonzero and defined by [14]

$$
u_{2}\left(x_{1}, x_{2}\right)=\frac{1}{\left(2 x_{1} R e\right)^{1 / 2}}\left[u_{1}\left(x_{1}, x_{2}\right) \int_{0}^{\eta} \tilde{T}(s) \mathrm{d} s-T\left(x_{1}, x_{2}\right) \int_{0}^{\eta} \tilde{u}_{1}(s) \mathrm{d} s\right]
$$

where $\eta$ denotes the so-called Blasius characteristic length, defined as

$$
\eta=\left(\frac{R e}{2 x_{1}}\right)^{1 / 2} \int_{0}^{x_{2}} \rho\left(x_{1}, s\right) \mathrm{d} s
$$

and $\tilde{T}, \tilde{u}_{1}$ denote the temperature and streamwise velocity as a function of the similarity coordinate. Eigenfunction perturbations derived from the LST are superimposed on the initial base flow, thus defining the initial state vector for unsteady flow simulations. These perturbations have the form

$$
v=\epsilon \operatorname{Re}\left(\psi\left(x_{2}\right) \exp \left[i\left(\alpha x_{1}-\omega t\right)\right]\right)
$$

where $\alpha$ is the wavenumber, $\omega$ the frequency, $\epsilon$ the initial perturbation amplitude and $\psi$ the complex eigenfunction vector. In the present case of spatial simulations, $\alpha$ is complex whereas $\omega$ is real. Hence, if $\operatorname{Im}(\alpha)<0$, the perturbation amplitude will grow exponentially in the streamwise direction. The flow is maintained by imposing the base flow together with the time-periodic perturbations at the inflow boundary.

\section{NUMERICAL METHODS}

In this section, the spatial discretization methods for the compressible Navier-Stokes equations developed in this paper are outlined. Specifically, the differential form of these equations can be written in the following conservative formulation:

$$
\partial_{1} U+\partial_{j} f_{j}(U)=0
$$

where the vector $U$ represents the conserved variables $\left(\rho, \rho u_{i}, e\right)^{\mathrm{T}}$ and $f_{j}$ the flux in the $x_{j}$ direction, consisting of the convective and the viscous contributions. It is important that the discretization can also be written in conservative form, and dissipation and dispersion errors are kept to a minimum. Best suited for this purpose are, for instance, spectral methods and high order central difference approximations. In view of an extension of the method to non-orthogonal grids in the 
future, we favour a finite difference approach. In this section two spatial discretization methods are described which are, respectively, second order and fourth order accurate on smooth grids.

The second order accurate discretization used here is a finite volume method, which is conservative, and can readily be formulated for an orthogonal non-uniform grid. The discretization for the convective terms is the cell vertex trapezoidal rule which is a weighted second order central difference. In vertex $(i, j)$ the derivative of a variable $f$ with respect to e.g. $x_{1}$ is defined as

$$
\left(\partial_{1} f\right)_{i, j}=\frac{s_{i+1, j}-s_{i-1, j}}{x_{1(i+1)}-x_{1(i-1)}}
$$

with

$$
s_{i j}=\frac{1}{2} \frac{\left(x_{2(j)}-x_{2(j-1)}\right)\left(f_{i, j}+f_{i, j-1}\right)+\left(x_{2(j+1)}-x_{2(j)}\right)\left(f_{i, j+1}+f_{i, j}\right)}{x_{2(j+1)}-x_{2(j-1)}} .
$$

The viscous terms contain second order derivatives which are approximated by the consecutive application of two first order numerical differentiation methods. In centre $(i, j)$ the discretization of $\partial_{1}^{2} f$ has the form

$$
\left(\partial_{1}^{2} f\right)_{i, j}=2 \frac{s_{i+\frac{1}{2} j}-s_{i-\frac{1}{2} j}}{x_{1(i+1)}-x_{1(i-1)}}
$$

with

$$
s_{i+\frac{1}{2} j}=\frac{1}{2}\left(\left(\partial_{1}^{\text {in }} f\right)_{i+\frac{1}{2} j-\frac{1}{2}}+\left(\partial_{1}^{\text {in }} f\right)_{i+\frac{1}{2} j+\frac{1}{2}}\right) .
$$

The 'inner' derivatives $\partial_{1}^{\text {in }} f$ are calculated with the same discretization rule as equation (11), but now applied to control volumes centred around vertices $(i+1 / 2, j+1 / 2)$ :

$$
\left(\partial_{1}^{\text {in }} f\right)_{i+\frac{1}{2} j+\frac{1}{2}}=\frac{g_{i+1, j+\frac{1}{2}}-g_{i j+\frac{1}{2}}}{x_{1(i+1)}-x_{1(i)}}
$$

with

$$
g_{i, j+\frac{1}{2}}=\frac{1}{2}\left(f_{i, j}+f_{i, j+1}\right)
$$

The scheme for the viscous terms prevents odd-even decoupling. Each of the above finite difference schemes for first order spatial derivatives in $(i, j)$ can be written in the following form:

$$
\left(\partial_{1} f\right)_{i, j}=\sum_{k=-n}^{k=n} w_{k} g_{i+k, j}
$$

with

$$
g_{i, j}=\sum_{k=-n}^{k=n} h_{k} f_{i, j+k}
$$

where $n=1$ for the above three-point second order approximation. We call $w$ differencing weights and $h$ averaging weights.

A fourth order scheme for the calculation of the convective terms can be formulated in the form (13) using a five-point molecule $(n=2)$. The weights can be computed by imposing certain requirements on the discretization scheme. We require the difference scheme to be exact for a set of basis polynomials, leading to

$$
\left(\partial_{1} f\right)_{i, j}=\frac{4}{3} \frac{\left(s_{i+1, j}-s_{i-1, j}\right)}{\left(x_{1(l+1)}-x_{1(i-1)}\right)}-\frac{1}{3} \frac{s_{i+2 j}-s_{i-2, j}}{\left(x_{1(i+2)}-x_{1(i-2)}\right)}
$$


Table 1. Prediction of temporal growth rates for the 2D and 3D instability modes using the fourth order method

\begin{tabular}{ccc}
\hline Resolution & 2D mode $\left(\times 10^{-2}\right)$ & 3D mode $\left(\times 10^{-2}\right)$ \\
\hline 48 & 0.20266544 & 0.08301932 \\
64 & 0.19708027 & 0.10828506 \\
96 & 0.19325113 & 0.11524572 \\
128 & 0.19237002 & 0.11608238 \\
192 & 0.19202539 & 0.11632751 \\
\hline
\end{tabular}

with

$$
s_{i, j}=\sum_{k=-2}^{k=2} h_{k} \int_{i, j+k}
$$

where the averaging weights $h_{k}$ read

$$
\begin{gathered}
h_{-2}=-\frac{1}{8} \frac{x_{2(j)}-x_{2(j-2)}}{x_{2(j+2)}-x_{2(j-2)}}, h_{2}=-\frac{1}{8} \frac{x_{2(j+2)}-x_{2(j)}}{x_{2(j+2)}-x_{2(j-2)}} \\
h_{-1}=\frac{1}{2} \frac{x_{2(j)}-x_{2(j-1)}}{x_{2(j+1)}-x_{2(j-1)}}, h_{1}=\frac{1}{2} \frac{x_{2(j+1)}-x_{2(j)}}{x_{2(j+1)}-x_{2(j-1)}} \\
h_{0}=\frac{5}{8} .
\end{gathered}
$$

It should be noticed that differentiation with respect to $x_{1}$ is carried out using differencing weights in the $x_{1}$ direction and averaging weights in the $x_{2}$ direction. This scheme is fourth order accurate for smooth grids. On a uniform grid the scheme reduces to the one used by Vreman et al. [15].

The weights for the second order derivatives of the viscous terms are computed analogously by employing four-point molecules to approximate each of the derivatives $\partial_{1}^{\text {in }} f$ and $\partial_{1}^{2} f$. The 'inner' derivative $\partial_{1}^{\text {in }} f$ is calculated in cell centres $(i+1 / 2 j+1 / 2)$ making use of the information on vertices $[i-1, \ldots, i+2, j-1, \ldots, j+2]$, whereas the second derivative $\partial_{1}^{2} f$ is computed in vertices $(i, j)$ using the derivatives $\partial_{1}^{\text {in }} f$ in cell centres $[i-3 / 2, \ldots i+3 / 2, j-3 / 2, \ldots, j+3 / 2]$. Based on the same grid resolution, the fourth order method requires approximately twice the computational effort of the second order method on a vector computer.

The above methods can also be formulated for 3D flow applications. To establish the order of accuracy in the 3D extension of the discretization methods quantitatively, we perform validation runs in the temporal setting in which we compare with predictions from linear stability theory (LST). In this case we adopt periodic conditions in the streamwise $\left(x_{1}\right)$ and spanwise $\left(x_{3}\right)$ directions. We consider a computational box of width equal to 30 in each of the directions and use a uniform grid in the streamwise and spanwise directions and a non-uniform grid which is clustered near the wall in the normal direction. For the initial state we use the compressible Blasius solution on which small amplitude perturbations as predicted by LST are superimposed. Two instability modes were considered: a 2D instability mode with temporal growth rate equal to $\omega_{i}^{(2 \mathrm{D})}=0.1919819 \times 10^{-2}$ and an oblique 3D mode with growth rate $\omega_{i}^{(3 \mathrm{D})}=0.116381175 \times 10^{-2}$. By performing a short time integration for each of these cases on a number of grids and monitoring the growth of the velocity fluctuations relative to the initial mean flow, the growth rate can be recovered from the simulation results. Specifically, we consider grids with $N^{3}$ grid cells where $N$ is taken from the Burlisch sequence $N=48,64,96,128,192$. The results of this resolution study for the fourth order method with Reynolds number based on the displacement thickness equal to 1000 and Mach number 0.5 are summarized in Table 1 . We observe an accurate prediction if the resolution is adequate. Moreover, a careful comparison of the results on the various grids indicates that the numerically obtained estimates for the growth rates converge to the LST prediction at a rate which is consistent with a fourth order method. Similar results have been obtained with the second order spatial discretization method, although the correspondence with the LST results is not as good as with the fourth order method.

The spatial discretization method presented here has already been successfully applied in three-dimensional simulations of laminar-turbulent transition in the temporal setting. Computations of the temporal mixing layer [16] and flow over a flat plate [17] established that, with 
sufficient resolution, a central differencing method for the convective terms and a 'staggered' discretization of the viscous terms yields reliable and accurate results. No artificial dissipation needs to be introduced. since the molecular dissipation provides a proper drain of the energy contained in the small scales in case the resolution is high enough to overcome a possible negative influence of aliasing errors. Alternatively, one may adopt 'kinetic energy conserving' schemes [18] which arise if a central differencing scheme is applied to the skew-symmetric form of the convective terms [19]. Such schemes prevent a divergence of kinetic energy but do not guarantee that other possible instabilities in a compressible solver will not occur (e.g. locally negative temperature). Our scheme discretizes the convective terms in the standard divergence form and, consequently, mass, momentum and total energy (kinetic plus internal energy) are conserved. At sufficient resolution the viscous terms make the numerical method stable.

The discretization near the boundaries is performed in the same manner as in the interior domain by creating dummy points outside the boundaries. Dummy variables are obtained through extrapolation of the interior variables. Second order and fourth order extrapolation polynomials are used for, respectively, the second order and fourth order finite difference schemes, except along the freestream boundary which always employs second order extrapolation. Furthermore, no conservation equation needs to be discretized along the wall. The condition $\partial_{2} p=0$ at the wall is discretized in a second order accurate way. These boundary treatments maintain a high order accuracy in the spatial discretization near the boundaries.

After the spatial discretization is performed, the system of partial differential equations (9) can be combined into a system of ordinary differential equations for the discrete state vector $u$ :

$$
\frac{\mathrm{d} u}{\mathrm{~d} t}=F(u)
$$

where $F$ is the discrete flux vector. This system of differential equations is solved using a four-stage compact-storage Runge-Kutta method. It performs within one time step $\Delta t$ :

$$
u^{(n)}=u^{(0)}+\alpha_{n} \Delta t F\left(u^{(n-1)}\right), \quad n=1,2,3,4
$$

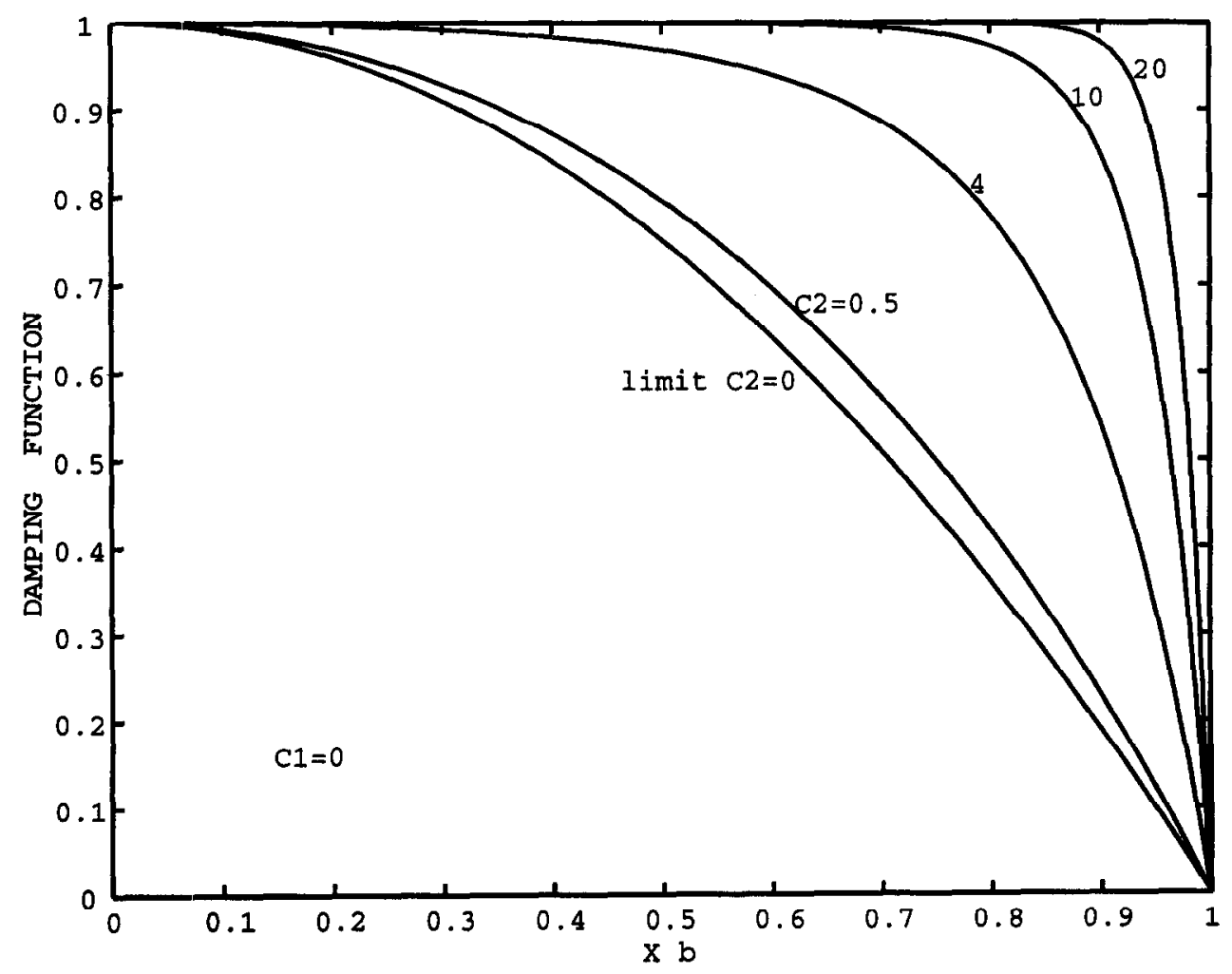

Fig. 2. Damping functions within the buffer domain using various values of $C_{2}$ at $C_{1}=0$. 


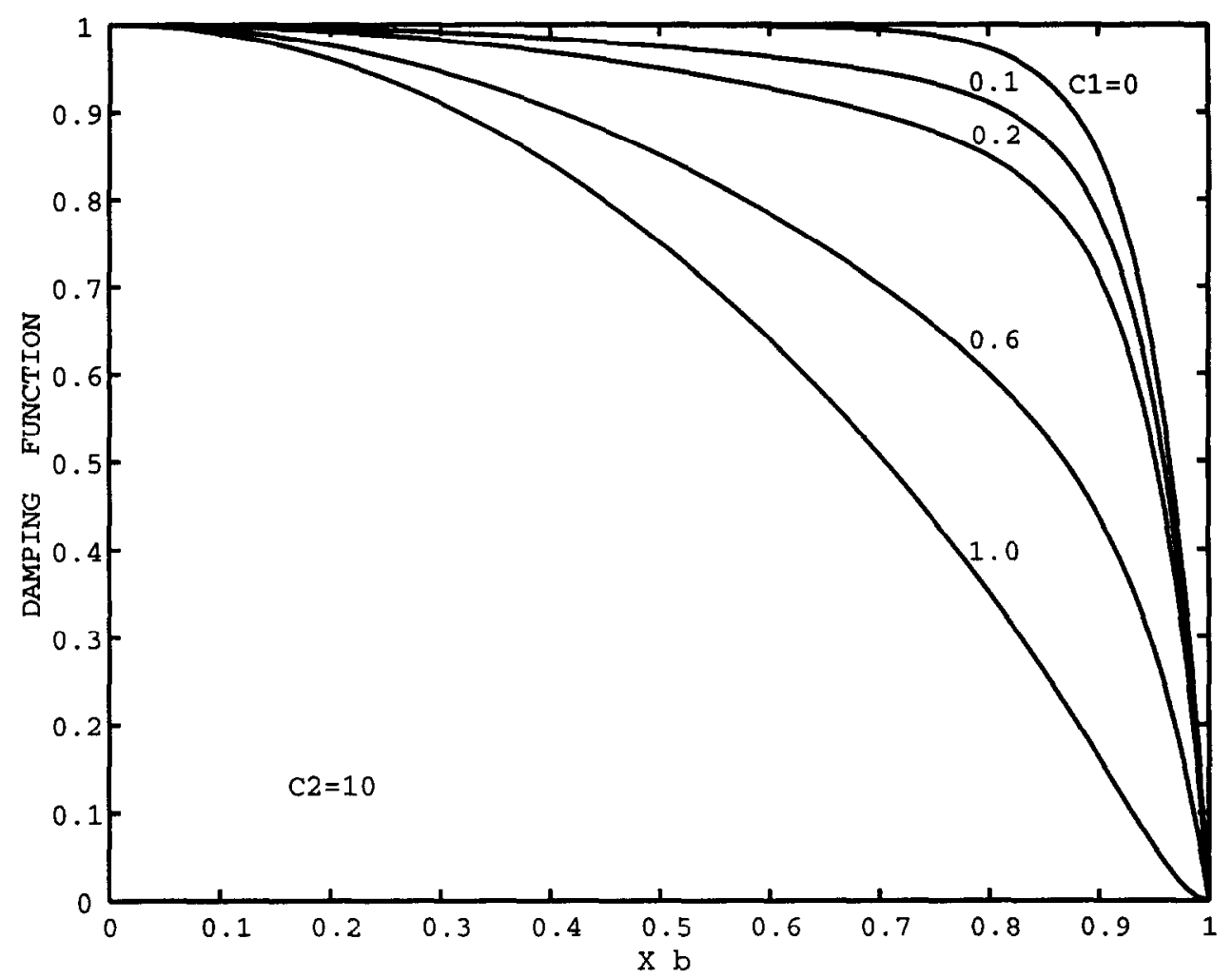

Fig. 3. Damping functions within the buffer domain using various values of $C_{1}$ at $C_{2}=10$.

with $u^{(0)}=u(t)$ and $u(t+\Delta t)=u^{(4)}$. With the coefficients $\alpha_{1}=1 / 4, \alpha_{2}=1 / 3, \alpha_{3}=1 / 2$ and $\alpha_{4}=1$ this yields a second order accurate time integration method [20].

\section{BOUNDARY CONDITIONS AND BUFFER DOMAIN TREATMENTS}

In this section, the specification of the artificial boundary conditions, i.e. the inflow, the outflow and the freestream boundary conditions, is described. In addition, a buffer domain treatment will be introduced. The number of physical boundary conditions required along the artificial boundaries is consistent with the well-posedness requirement for the hyperbolic system, as derived by Strikwerda [7]. These boundary conditions are provided by information about the external flow, adjacent to the boundaries. If this external flow information is not known accurately, which can occur in the case of subsonic outflow, at the freestream and outflow boundaries, a so-called non-reflection condition serves to provide the boundary condition. At the inflow boundary, we impose the compressible Blasius solution with linear perturbations added as the external information. More specific descriptions are given in the following subsections.

\subsection{Inflow boundary conditions}

At the inflow boundary of subsonic flow, the required external information is provided by imposing the velocity components and the temperature. The pressure at the inflow boundary is determined by using information from the interior domain. Several alternatives can be applied to specify the inflow pressure: extrapolation from the interior pressure or using a characteristic method as proposed by Rudy-Strikwerda [4] or Poinsot-Lele [6]. Numerical experiments show, however, that the characteristic method yields small oscillations in the boundary layer near the inflow boundary. This can be explained from the fact that the characteristic method is derived from inviscid flow theory. For this reason, we use the extrapolation variant which is specified in such a way that it is zeroth order outside the boundary layer and gradually becomes second order near 
the wall. Further increasing the order of extrapolation in the boundary layer can trigger instabilities near the inflow boundary. More specifically

$$
\begin{gathered}
T=\hat{T}\left(x_{2}, t\right) \\
u_{i}=\hat{u}_{i}\left(x_{2}, t\right), \quad i=1,2 \\
p=b^{(1)}\left(x_{2}\right) p^{(1)}\left(x_{2}, t\right)+b^{(2)}\left(x_{2}\right) p^{(2)}\left(x_{2}, t\right)+b^{(3)}\left(x_{2}\right) p^{(3)}\left(x_{2}, t\right)
\end{gathered}
$$

where $p^{(1)}, p^{(2)}$ and $p^{(3)}$ are the pressures at the first, second and third interior points in the streamwise direction, respectively, and $b^{(1)}, b^{(2)}$ and $b^{(3)}$ are the extrapolation weights. Outside the boundary layer $b^{(1)}=1$ and $b^{(2)}=b^{(3)}=0$, whereas at the wall $b^{(1)}=-b^{(2)}=3$ and $b^{(3)}=1$. Inside the boundary layer $b^{(i)}=\left(1-\bar{u}_{1}\right) b_{\mathrm{w}}^{(i)}+\bar{u}_{1} b_{\mathrm{o}}^{(i)}$, where $i=1,2,3$; the subscripts $\mathrm{w}$ and o denote the value at the wall and outside the boundary layer, respectively, and $\bar{u}_{1}$ is the streamwise velocity component of the compressible Blasius solution. $\hat{T}$ and $\hat{u}_{i}$ describe the behaviour of the temperature and the velocity at the boundary. In time-dependent simulations, these consist of the compressible Blasius profiles with perturbations added following from linear stability theory. The condition on $p$ allows the pressure at the inflow to vary in response to the disturbances originating from the interior flow.

For supersonic flow all variables, $T, u_{1}, u_{2}$ and $p$, which consist of a base flow with linear eigenfunction perturbations added are imposed at the inflow boundary. The subsonic flow part near the wall should be treated using the subsonic inflow boundary conditions. However, experiments show that when we consider high supersonic Mach numbers, the supersonic boundary conditions can be used throughout the inflow boundary as the region of subsonic layer near the wall is small.

\subsection{Outflow and freestream boundary conditions}

The outflow boundary condition used in this study has been proposed by Poinsot and Lele [6]. In this treatment the convective flux in the streamwise direction is approximated in terms of the amplitude variation of characteristic waves, whereas the formulation of the convective flux in the

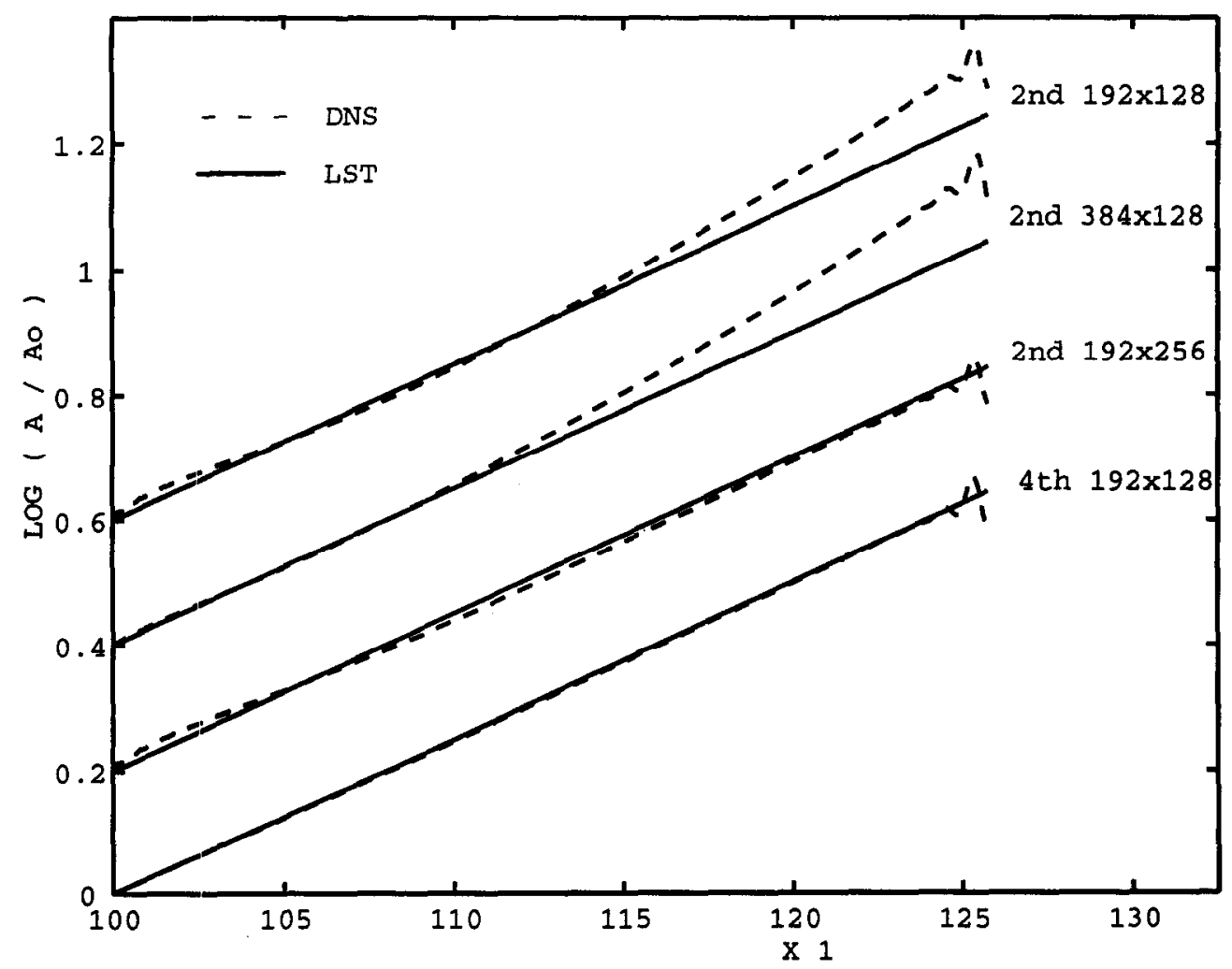

Fig. 4. Comparison of the growth of disturbance amplitude, taken from the $u_{1}^{\prime}$ component, between the second order scheme on various grids and the fourth order scheme on the coarsest grid. 


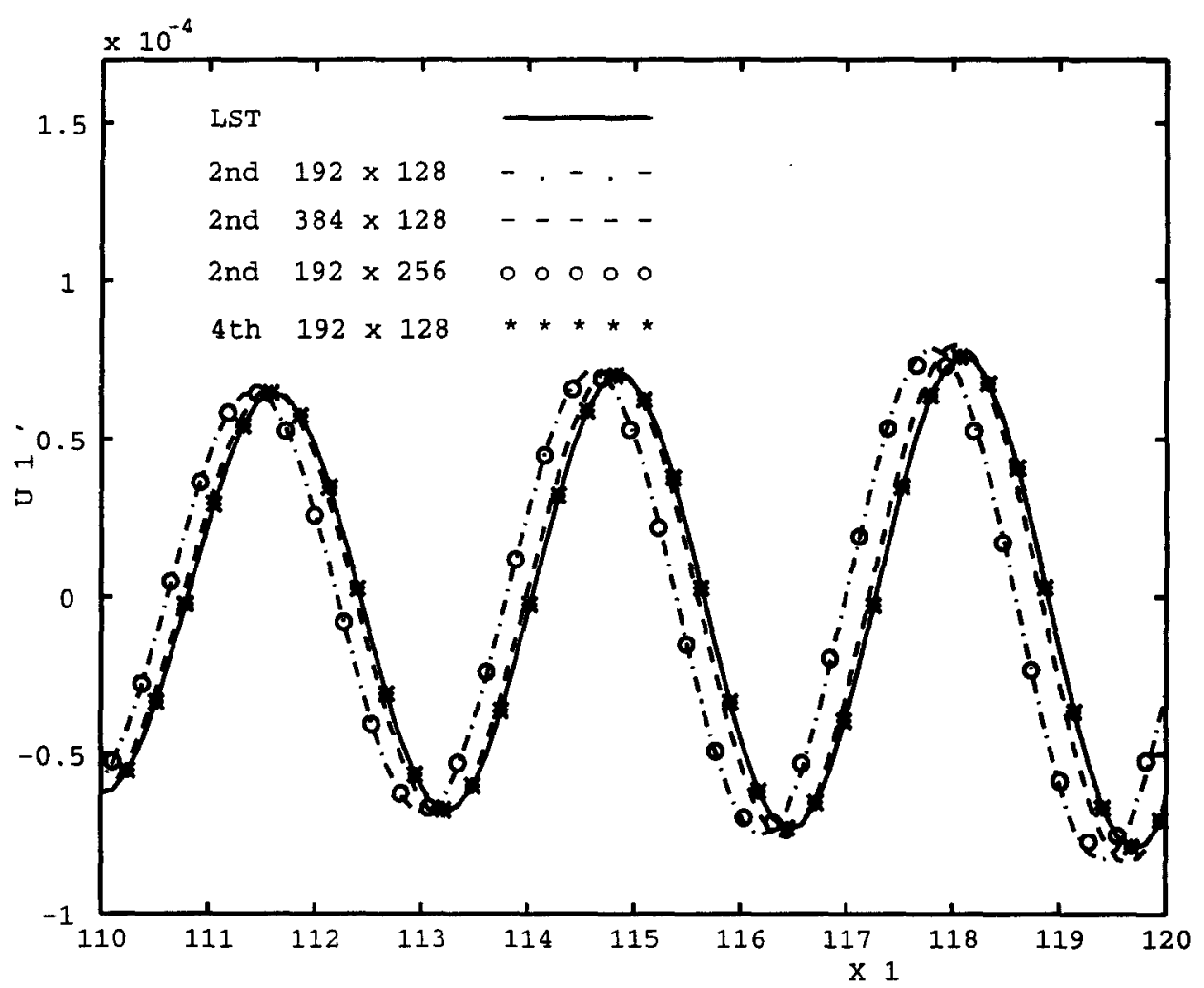

Fig. 5. The same comparison as in Fig. 4 for the development of $\mathbf{T}-\mathbf{S}$ waves in the streamwise direction.

normal direction remains unchanged. The amplitude variations are in turn expressed as a linear combination of the first derivatives of all dependent variables with respect to the streamwise coordinate. They consist of four components corresponding to four characteristic waves which travel with velocities $u_{1}-c, u_{1}, u_{1}$ and $u_{1}+c$, where $c$ is the local speed of sound given by $c^{2}=\gamma p / \rho$. The boundary conditions are implemented by imposing certain demands on the amplitude variation. In a supersonic flow in which $u_{1}>c$, all characteristic waves travel in the streamwise direction. In this case, the amplitude variation of all these waves can be calculated using the information upstream of the outflow boundary. Hence no specific demand is imposed on the amplitude variations in the supersonic case. In a subsonic flow, however, one characteristic wave travels upstream. The amplitude variation of this particular wave is not known since we have no information from downstream of the outflow boundary, which is outside the computational domain. In this case a so-called perfectly non-reflecting condition is imposed which requires that there should be no variation in the amplitude of the incoming wave. In addition to the convective condition, two viscous relations $\partial_{1} \tau_{12}=0$ and $\partial_{1} q_{1}=0$ are imposed, complementing the required number of boundary conditions. The perfectly non-reflecting condition and the viscous relations are aimed at minimizing wave reflections at the outflow boundary.

The same boundary conditions as above are used along the freestream boundary. The only difference is that it is now applied in the normal direction instead of the streamwise direction and that no viscous relations are imposed in view of the fact that the freestream flow is almost purely convective. These fully inviscid boundary conditions were originally proposed by Thompson [5].

\subsection{Buffer domain treatments}

In the vicinity of the outflow boundary a buffer domain is optionally employed. The objective of the buffer region is to prevent reflection of the fluctuations which have built up inside the upstream part of the computational domain as a consequence of physical instabilities in the flow. Common ways to achieve this goal are a considerable increase of the viscosity [9], a gradual change of the governing equations into a parabolic system $[9,11,12]$ and removing the disturbance 
component from the convective velocity $[11,12]$. The last method was originally applied by Streett and Macaraeg [12] for incompressible flow.

Experiments indicate that the larger the viscosity increase the more effectively the buffer damps the fluctuations. However, for calculations with explicit time stepping, the increase of viscosity yields a considerable limitation in the allowable time step. This viscous limitation on the time step becomes rapidly more restrictive than the convective limitation. Consequently, this viscous time step restriction deteriorates the overall efficiency of the numerical method. The technique of applying the parabolizing procedure has only a gradual effect and consequently requires the use of quite extended, and hence costly, buffer domains in order to be effective.

We propose a more efficient method in which the disturbances of all the solution components are gradually reduced to zero within the buffer domain by directly multiplying the disturbances with an appropriate damping function. This gives a better result than the removal of disturbances only from the convective velocity, even without parabolizing the governing equations. This method requires only a short buffer domain to damp wave reflections at the outflow boundary, as will be shown in the computational results. In this direct approach we need a reference flow, relative to which the disturbances can be damped within the buffer domain. The reference profile used here is the initial base flow in the case of parallel flow and a time averaged flow in the case of nonparallel flow, which is further described in Section 5.3. The amplitudes of the T-S waves are gradually reduced by multiplying the disturbances of all components with a damping function which gradually decreases from 1 to 0 . Hence, near the boundary all fluctuations are effectively removed from the signal and the flow is relaminarized. This approach can be described in the following formula:

$$
U=U_{\mathrm{ref}}+\zeta\left(x_{1}\right)\left(\tilde{U}-U_{\mathrm{ref}}\right)
$$

where $U=\left(\rho, \rho u_{i}, e\right)^{\mathrm{T}}, U_{\text {ter }}$ is the similarity solution to the boundary layer equations, $\tilde{U}$ is the solution calculated without applying the buffer treatment, and $\zeta$ is a damping function. The damping given in equation (22) is applied to every stage of the Runge-Kutta scheme. In the damping function we use a buffer domain coordinate $x_{\mathrm{b}}$ which is defined as

$$
x_{\mathrm{b}}=\frac{x_{1}-x_{\mathrm{s}}}{x_{\mathrm{e}}-x_{\mathrm{s}}}
$$

where $x_{\mathrm{s}}$ and $x_{\mathrm{e}}$ are the values of $x_{1}$ at the beginning and the end of the buffer domain, respectively. Thus $x_{\mathrm{b}}$ ranges from 0 to 1 , marking, respectively, the beginning and the end of the buffer domain. The damping function $\zeta$ is required to satisfy the following constraints:

$$
\begin{gathered}
\zeta(0)=1 \\
\frac{\partial \zeta}{\partial x_{\mathrm{b}}}(0)=0 \\
\frac{\partial \zeta}{\partial x_{\mathrm{b}}}\left(x_{\mathrm{b}}\right) \leq 0 \text { if } x_{\mathrm{b}}>0 \\
\zeta(1)=0 .
\end{gathered}
$$

The first constraint lets disturbances enter the buffer domain at the original level of amplitude, the second constraint prevents discontinuity at the beginning of the buffer domain, the third constraint forces $\zeta$ to decrease monotonically and the fourth constraint guarantees a zero disturbance amplitude at the outflow boundary. The reduction of disturbances is carried out at every stage and hence has a cumulative effect. Already reduced disturbances at a certain time will be multiplied again by the damping function at the next stage, and so on. Hence, the amplitude of the disturbances decreases more rapidly than the decrease of the damping function. However, this successive reduction of the amplitude will not result in a sudden suppression of the amplitude of the disturbances. Numerical experiments show that a balance arises between the growth of disturbances fed by incoming disturbances from upstream of the buffer domain and the damping of the disturbances within the buffer domain. We next proceed with specifying the damping function $\zeta$ in a few steps. The simplest damping function which satisfies the above four constraints, CAF 26/7 C 
$\zeta\left(x_{\mathrm{b}}\right)=1-x_{\mathrm{b}}^{2}$, results in a too abrupt decrease of the disturbance amplitudes. To achieve a more gradual decrease, we use

$$
\zeta=\left(1-C_{1} x_{\mathrm{b}}^{2}\right)\left(1-\frac{1-\mathrm{e}^{C_{2} x_{\mathrm{b}}}}{1-\mathrm{e}^{C_{2}}}\right) \text { with } 0 \leq C_{1}<1, C_{2}>0 .
$$

By varying $C_{1}$ and $C_{2}$, different shapes of the damping function can be obtained. As an illustration, the shape of the damping function for various values of $C_{1}$ and $C_{2}$ is depicted in Figs 2 and 3 . By selecting appropriate values of $C_{1}$ and $C_{2}$ we can control the reduction rate of the disturbances. Numerical experiments lead to $0 \leq C_{1} \leq 0.1$ and $10 \leq C_{2} \leq 20$ as appropriate. Notice that $C_{1}=0$ is allowed, since the second term in equation (24) itself satisfies all four constraints (23).

As a final step in the specification of the buffer function, we make it insensitive to the mesh size. We note that the result of the buffer procedure for a certain flow configuration depends on the number of time steps per disturbance period, since the buffer function is applied after every Runge-Kutta stage. Hence, when we use a higher grid density, the buffer procedure is applied more frequently. Besides, the number of time steps per period depends also on the flow conditions $\left(M_{x}\right.$, $R e$, etc.). In order to make the buffer procedure independent of the number of time steps per disturbance period, we employ a damping function $\tilde{\zeta}$ given by

$$
\tilde{\zeta}=\zeta^{c_{3} / N}
$$

where $\tilde{C}_{3}$ is a tuning parameter and $N$ is the number of time steps per disturbance period, which is unknown prior to the simulation. $N$ can be approximated by $N=T / \Delta t$, where $T$ denotes one period of the inflow perturbation and $\Delta t$ the allowable time step. Since $T=2 \pi / \omega$, where $\omega$ is the circular frequency of the disturbance, this leads to

$$
\tilde{\zeta}=\zeta^{C_{3} \omega \Delta t}
$$

where $\zeta$ is given by equation (24) and $C_{3}$ is a tuning parameter, which is kept constant throughout the simulations.

Our numerical tests have shown that this direct approach is substantially more efficient than the

A)

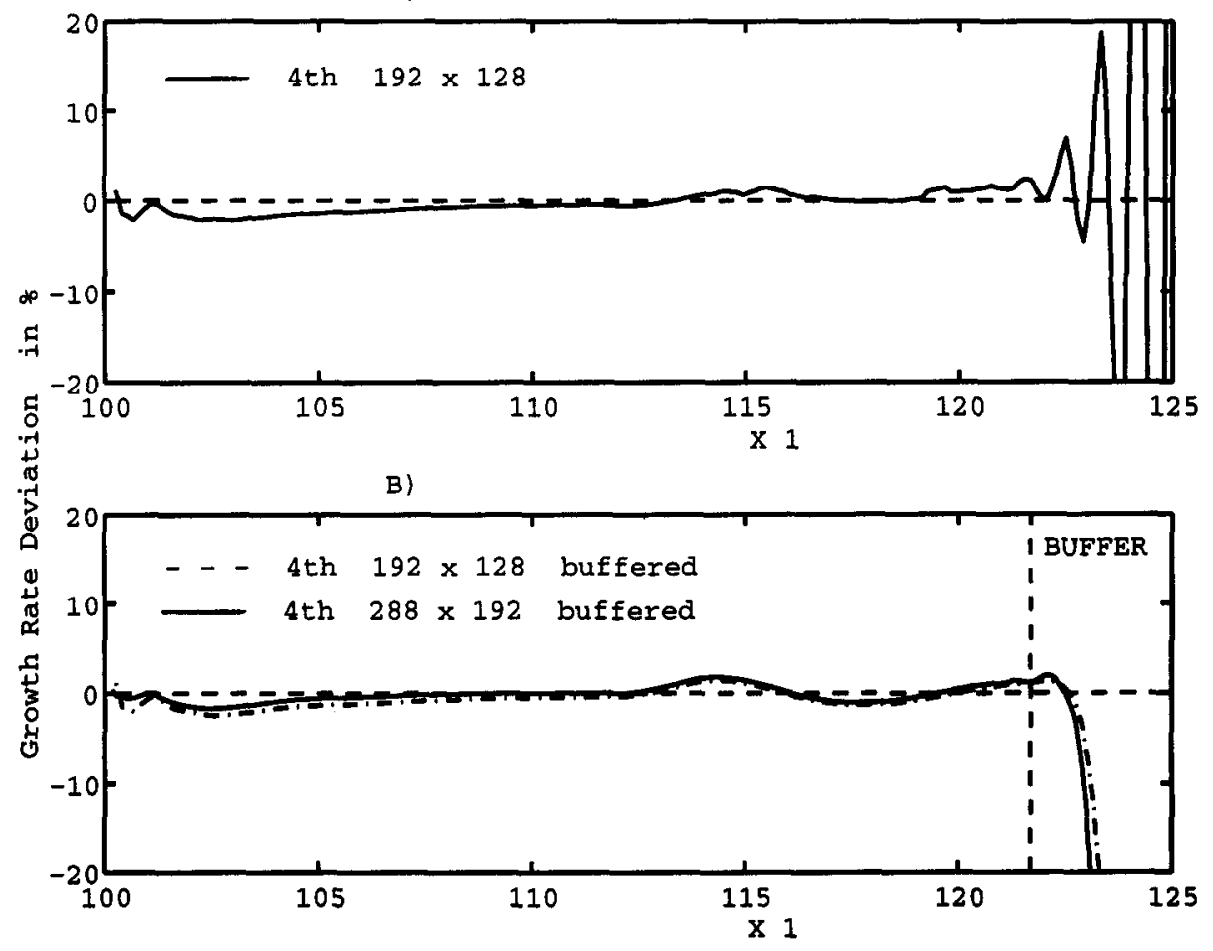

Fig. 6. Growth rate deviation in $u_{1}^{\prime}$ component: (A) without buffer domain; (B) with buffer domain. The result with a finer grid in the $x_{1}$ direction is also presented. 


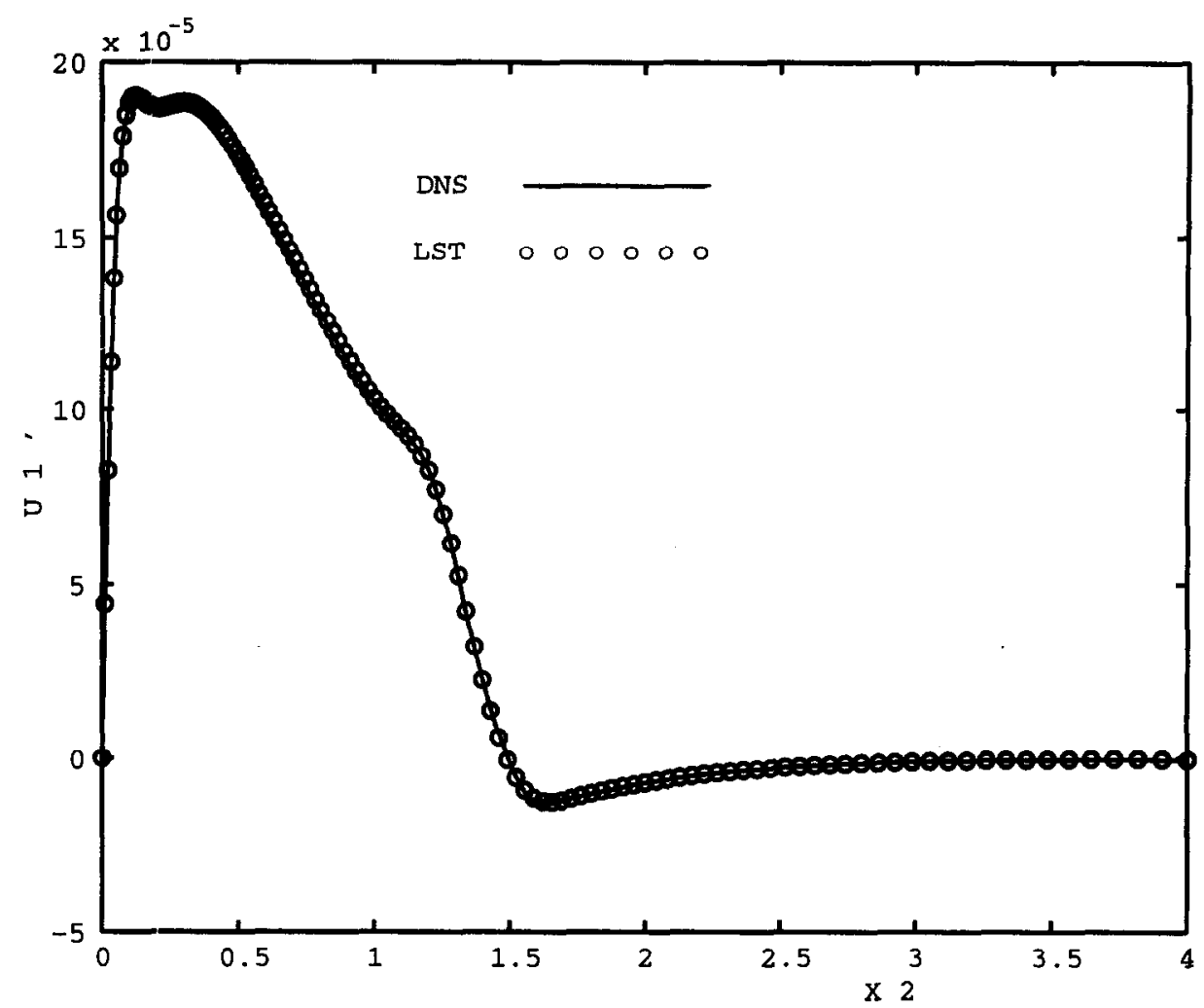

Fig. 7. Comparison of the disturbance profile of $u_{1}^{\prime}$ fundamental wave at $x_{1}=121.29$ between DNS and LST.

approach of increasing viscosity with respect to both computational effort and reflection properties. The validation of the buffer domain approach including the calibration of the tuning parameters and the investigation of its performance for various buffer domain lengths and various lengths of the physical domain is presented in Section 5. We have applied this buffer domain technique to more complex flows, such as separated flows [21] and unsteady shock boundary layer interaction, and obtained satisfactory results. It should be noted that the reference flow $U_{\text {ref }}$ depends on the flow under consideration, whereas the damping function is generally applicable.

\section{COMPUTATIONAL RESULTS}

In this section, some computational results are presented which illustrate the effectiveness of the approach followed. In particular, we consider parallel and nonparallel base flow with small perturbations and nonparallel base flow with large perturbations. The simulations of parallel base flow with small perturbations are performed in the subsonic and the supersonic regime with Mach number 0.5 and 4.5 , respectively to study the effect of essentially different inflow and outflow characteristics on the flow. The calculations of nonparallel flow in the linear and nonlinear regimes are performed at Mach number 0.5. In the linear regime small disturbances are introduced at the inflow boundary. Nonlinear effects become appreciable by increasing the disturbance amplitude. The results of calculations with parallel base flow are compared with the LST results, whereas the nonparallel flow calculations are confronted with the solution of PSE. For discussions regarding LST and PSE one can refer to Refs [22, 23], respectively. All results are presented after a statistically stationary state is attained. This can be verified by monitoring the development of the growth rate with time. The computational time needed is typically twice the amount of time required for the leading wavefront to pass from the inflow to the outflow boundary.

\subsection{Parallel base flow with small perturbations at Mach 4.5}

First, it is important to verify the accuracy of the discretization scheme and the performance of inflow and outflow boundary treatments with small disturbances. Therefore, a simulation of unsteady flow has been performed at $M_{x}=4.5$ and $T_{x}=61.15 \mathrm{~K}$, in which time dependent 
perturbations are added to a parallel base flow. This flow falls within a second mode instability region (Mack mode [23]). Since a flow over a flat plate is physically nonparallel, forcing terms are introduced in the right-hand side of the momentum and energy equations in the Navier-Stokes system to keep the flow parallel. Denoting the forcing term of the $u_{1}$ and $u_{2}$ momentum equations and the energy equation by $F_{1}, F_{2}$ and $F_{\mathrm{e}}$ respectively:

$$
\begin{gathered}
F_{1}=-\partial_{2}\left(\frac{\mu}{R e} \partial_{2} \bar{\mu}_{1}\right) \\
F_{2}=0 \\
F_{\mathrm{e}}=-\partial_{2}\left[\bar{u}_{1} \frac{\mu}{R e} \partial_{2} \bar{u}_{1}+\bar{q}_{2}\right]
\end{gathered}
$$

where $\bar{u}_{1}$ and $\bar{q}_{2}$ are the streamwise velocity and the wall normal heat flux of the mean initial field, respectively.

The present physical parameters have also been used by Guo et al. in Ref. [3]. Specifically, the reference Reynolds number is $R e=8000$. The similarity solution at $x_{1}=100$ is used as the parallel base flow. This value of $x_{1}$ marks the position of the inflow boundary. Eigenfunction perturbations derived from compressible linear stability theory are added to the base flow defining the total initial flow. The circular frequency of the linear perturbations is $\omega=1.766$. The compressible linear stability theory provides an eigenvalue:

$$
\alpha=\alpha_{\mathrm{R}}+\mathrm{i} \alpha_{1}=1.94247-\mathrm{i} 0.02503
$$

which is an unstable mode. The Tollmien-Schlichting waves for the perturbations, travelling in the streamwise direction, can be described according to equation (8), where the value of $\epsilon$ used here is $10^{-4}$. In this test case of parallel flow the real and imaginary parts of the eigenfunctions, $\psi_{\mathrm{R}}^{\mathrm{r}}$ and

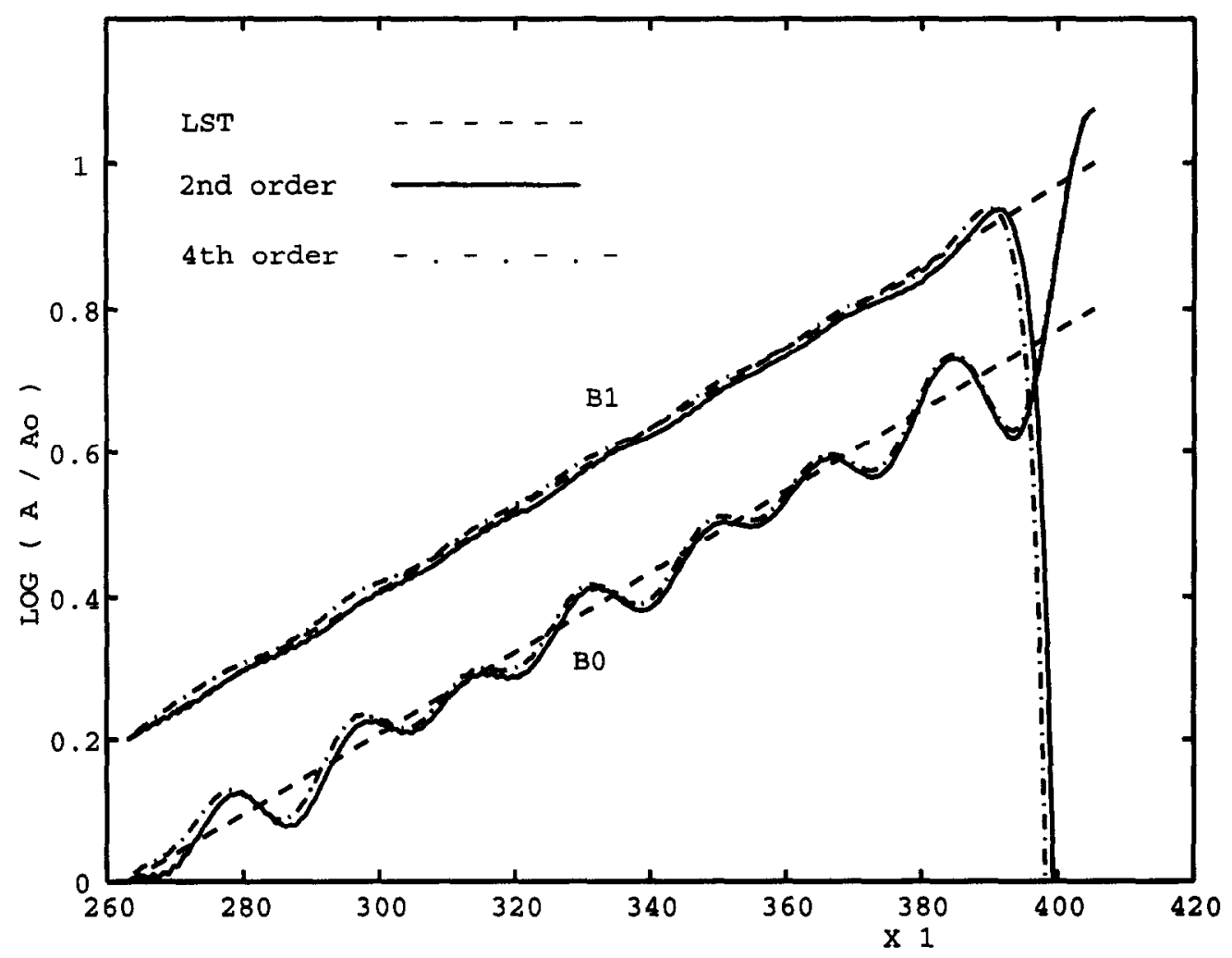

Fig. 8. Comparison of the amplitude growth taken from $u_{1}^{\prime}$ between treatment without buffer domain (BO) and with buffer domain (B1) using the second order scheme and the fourth order scheme. 


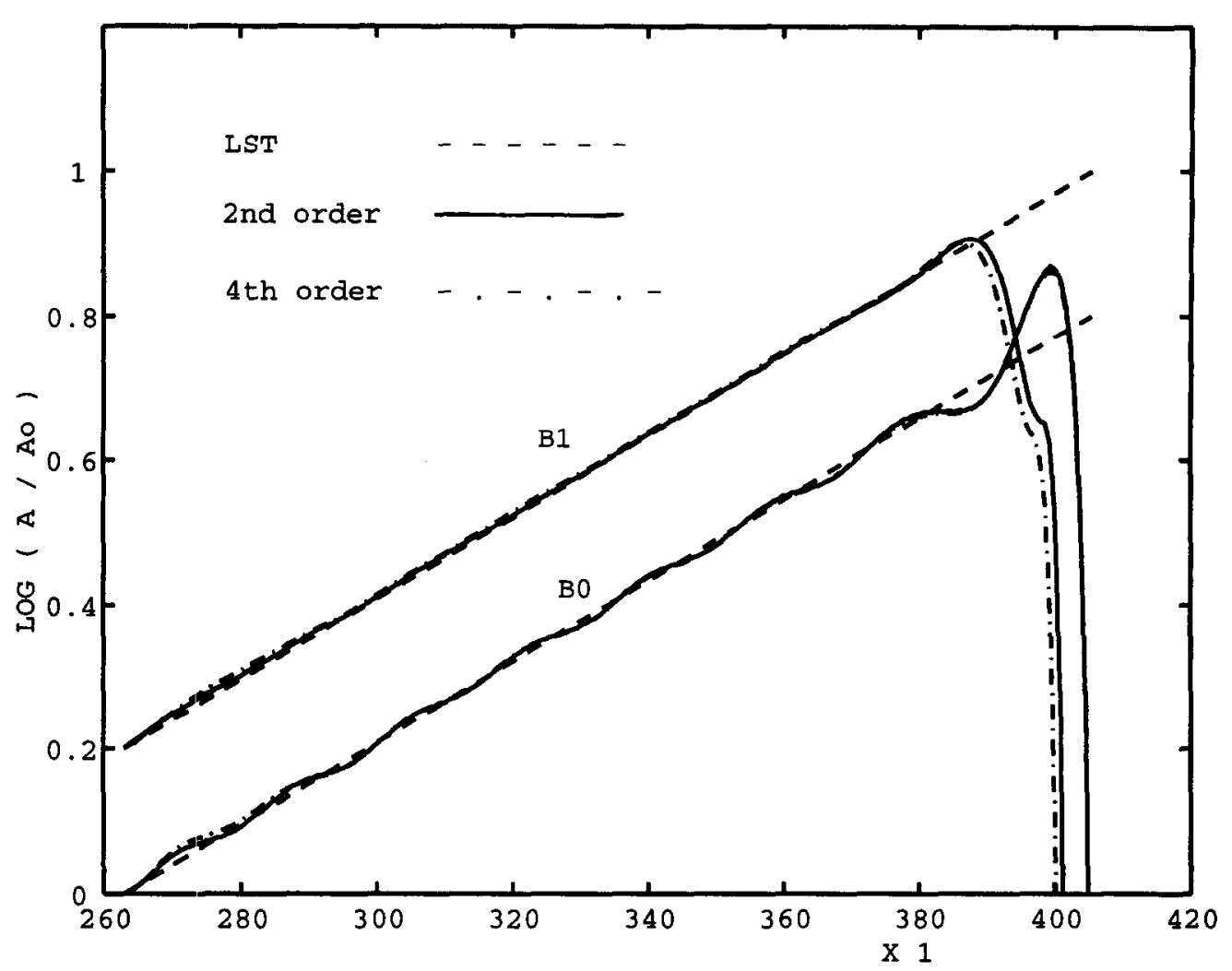

Fig. 9. The same comparison as in Fig. 8 for the amplitude growth of $u_{2}^{\prime}$.

$\psi_{1}^{r}$, are only dependent on $x_{2}$. It can easily be derived that the amplitude of a disturbance component $v_{i}$ grows in the $x_{1}$ direction according to the following expression:

$$
R_{i}=\epsilon \mathrm{e}^{-\gamma_{\mathrm{l}} \mathrm{r}_{1}} \sqrt{\left(\psi_{\mathrm{k}}^{\mathrm{r}}\right)^{2}+\left(\psi_{\mathrm{i}}^{\mathrm{i}}\right)^{2}}
$$

and since $\psi_{\mathrm{R}}$ and $\psi_{1}$ are constant in the $x_{1}$ direction, the growth of the amplitude is exponential in $x_{1}$. This does not hold when we consider nonparallel flows, as will be illustrated in Section 5.3.

The length of the computational domain is selected to be eight T-S wavelengths, of which the last is optionally used as a buffer domain. The height of the computational domain is $L_{2}=4$. We first use the second order scheme and a grid with $192 \times 128$ points in, respectively, the streamwise and the normal direction. Each T-S wave is thus represented by 24 points. The grid is uniform in the $x_{1}$ direction and stretched in the $x_{2}$ direction according to $x_{2}=L_{2} A y /(1+A-y)$, where $A$ is a stretching parameter and $0 \leq y \leq 1$, uniformly distributed. The grid becomes more uniform with increasing $A$. An appropriate value of $A$ in this test case is 0.5 , leading to the stretching ratio $\Delta x_{2 \max } / \Delta x_{2 \min }=8.8$. The maximum aspect ratio of the grid is $\Delta x_{1} / \Delta x_{2}=12.87$. The number of time steps per period is about 750 . The calculations are performed without buffer domain. The growth of the disturbance amplitude deviates from the LST result increasingly in the streamwise direction, as shown at the top of Fig. 4 (in this figure, the lines corresponding to the different grids and schemes have been shifted relative to each other to show the differences more clearly). The phase of the T-S waves deviates from the LST result as well, as depicted in Fig. 5. The disturbance data is taken from the $u_{1}^{\prime}$ component at a location near the maximum amplitude. The equivalent amplitude growth and T-S waves calculated from other components of the solution are quite similar to those of $u_{1}$. Addressing the deviations to insufficient grid density, we increase the grid density in the $x_{1}$ direction up to $384 \times 128$ grid points. Although the phase is now in better agreement with the LST result, this does not improve the amplitude growth. On the other hand, increasing the grid density in the $x_{2}$ direction $(192 \times 256$ grid points) yields a better amplitude growth but the deviation in the phase is about as large as with the original grid. From this knowledge it is apparent that the second order spatial discretization scheme requires a very fine grid, with at least $384 \times 256$ grid points, to achieve acceptable accuracy. In order to yield more 
accurate results with a moderate grid density, we employ the fourth order spatial discretization scheme as described in Section 3. Using the original grid $192 \times 128$, this scheme produces much better results than the second order scheme on finer grids. Both the amplitude growth and the T-S wave phase of the fourth order scheme are in excellent agreement with the LST results, as shown in, respectively, Figs 4 and 5. The number of time steps per period for the fourth order method on this coarse grid is around 1500 . For a comparable accuracy, the second order method requires the grid $384 \times 256$, which has four times as many points as the grid $192 \times 128$. In combination with the fact that the fourth order method requires more calculation time on a given grid than the second order method, it follows that the fourth order method needs half the computational effort to achieve the same accuracy.

Figure 6(A) shows the relative deviation of the local growth rates from the LST spatial growth rate, $-\alpha_{i}$, using the fourth order method. This parameter is critical for the performance of the inflow and outflow boundary conditions. The growth rates are calculated from the first order derivative with respect to $x_{1}$ of the local amplitude growths using a central difference scheme. The maximum deviation of the growth rates is about $2.5 \%$ over $87 \%$ of the domain. Strong oscillations originating from the outflow boundary conditions are restricted within the last $13 \%$ of the domain. Although we do not employ any buffer domain treatment in these calculations, the maximum deviation compares favourably with the work reported in Ref. [3]. Especially near the inflow boundary, the error is kept to a minimum thanks to the accurate numerical treatment near this boundary. This is confirmed by varying the degree of the extrapolation polynomials used at the boundaries from the default value 4 . Lower degree polynomials yield larger errors near the inflow and outflow boundaries. On the other hand, increasing the polynomial degree to more than 5 can lead to numerical instabilities at the boundaries. Small oscillations which are appreciable along the second half of the domain are damped when the buffer domain treatment is employed (the buffer domain validation is discussed in the next section), as shown in Fig. 6(B). However, small waves remain present along the second half of the domain. Increasing the resolution in the streamwise direction decreases the errors along the first half of the domain. Varying the length of the

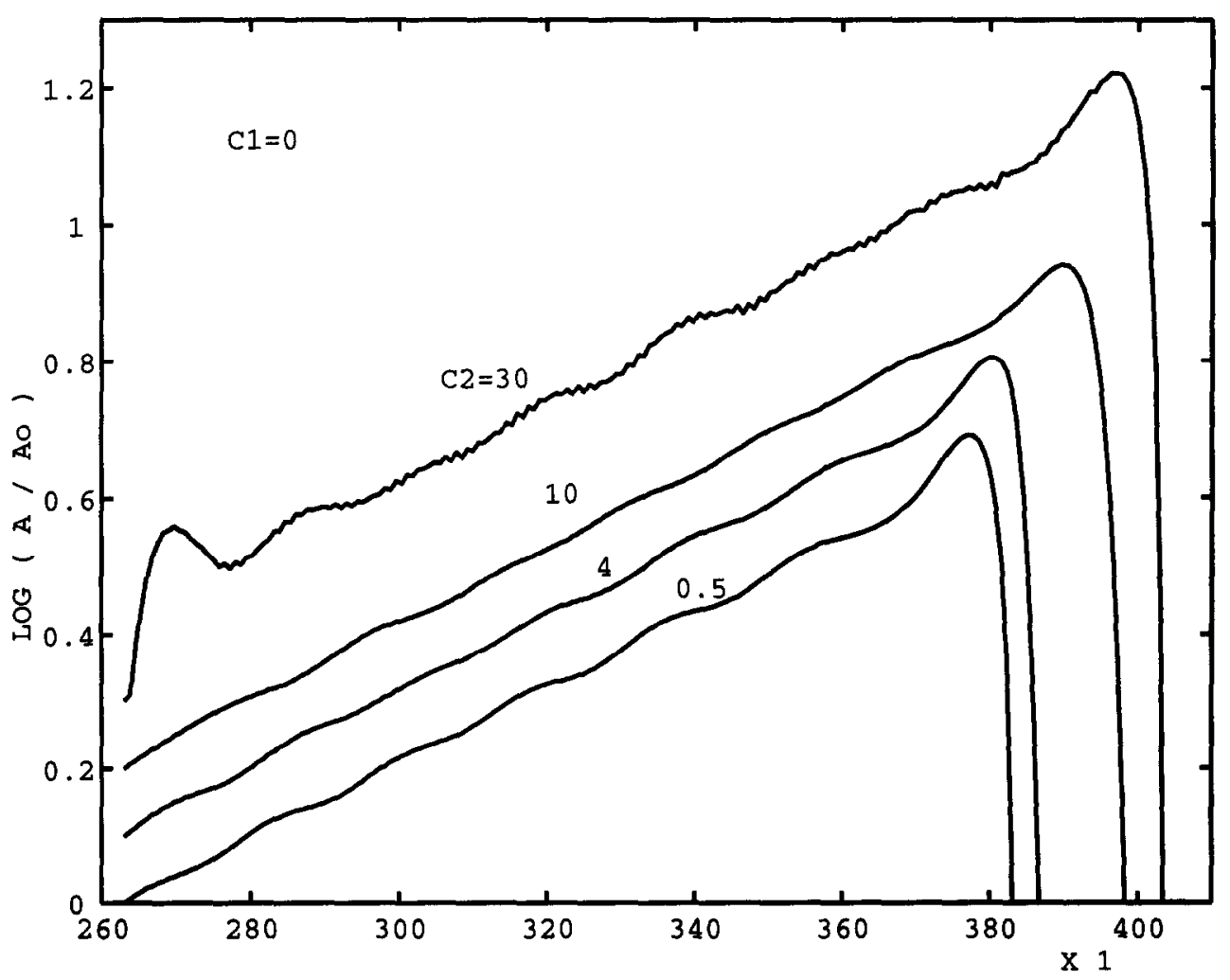

Fig. 10. Comparison of the amplitude growth, taken from $u_{1}{ }^{\prime}$, at various values of buffer parameter $C_{2}$ with $C_{1}=0$. 


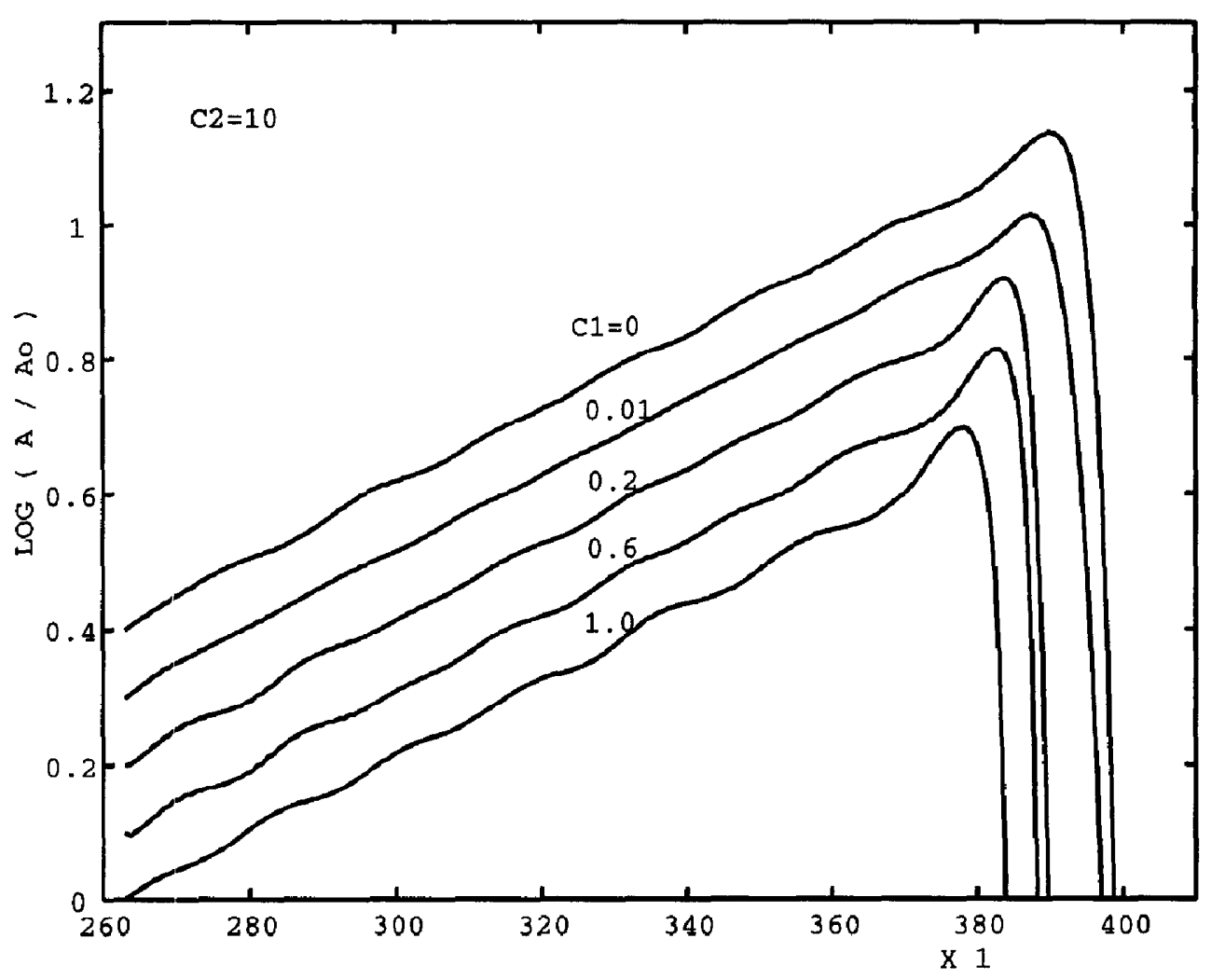

Fig. 11. Comparison of the amplitude growth, taken from $\boldsymbol{u}_{1}{ }^{\prime}$, at various values of buffer parameter $C_{1}$ with $C_{2}=10$.

computational domain indicates that the small waves are residual standing waves originating from the interaction between the inflow and outflow boundaries. Excellent agreement between the fundamental wave for $u_{1}$ at a certain streamwise position and the corresponding LST result is shown in Fig. 7. The streamwise position is selected such that the comparison is made in the neighbourhood of a local wave extremum. Equivalent results are produced by considering other components.

From these results we learn that the fourth order spatial discretization requires less computational effort than the second order method for a comparable accuracy. Furthermore, the inflow and outflow boundary treatments perform well for high supersonic flows. The outflow boundary exhibits only a small upstream influence and hence employing a buffer domain is not essential in order to damp wave reflections. The good performance of the outflow boundary conditions results apparently from the physics of the flow passing through the outflow boundary. In this flow application, most of the flow passing through the outflow boundary is supersonic and only a small part near the wall is subsonic. Therefore, the source of upstream influences is restricted near the wall. Flow simulations at a subsonic reference Mach number provide a more difficult test of the inflow/outflow boundary treatments and the buffer domain. This will be considered in the next sections.

\subsection{Parallel base flow with small perturbations at Mach 0.5}

Similar calculations as in the previous section are performed at a reference Mach number of 0.5 . The Reynolds number of the simulation is supercritical, equal to 875. The circular frequency $\omega$ of the linear perturbations is 0.1 . Compressible linear stability theory provides an eigenvalue:

$$
\alpha=\alpha_{\mathrm{R}}+\mathrm{i} \alpha_{1}=0.2649-\mathrm{i} 0.005246
$$

which is an unstable mode.

The length of the computational domain is equivalent to seven T-S waves, of which the last wavelength is optionally reserved for a buffer domain. The waves are represented on a grid with 


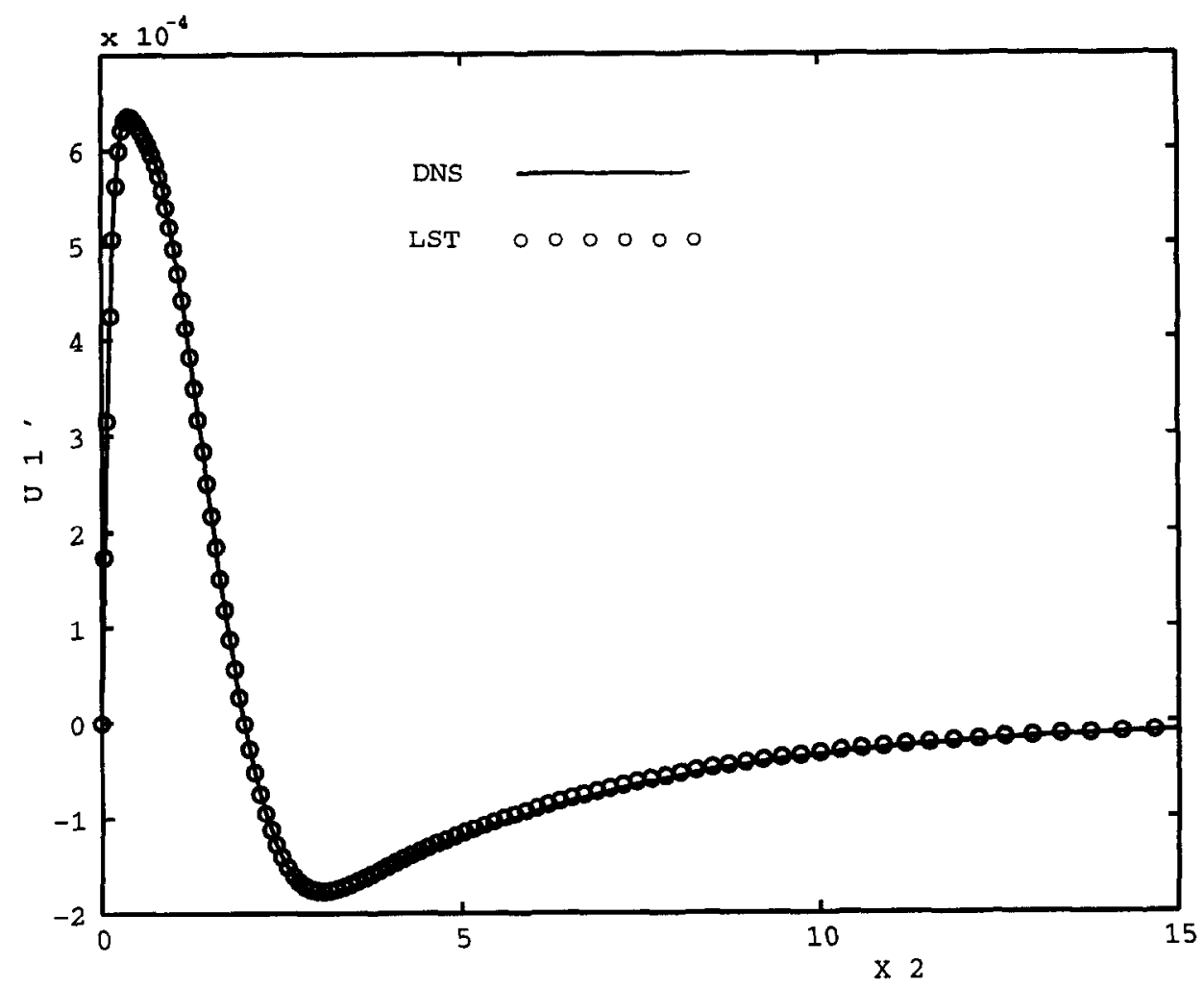

Fig. 12. Comparison between DNS and LST of the disturbance profile of the $u_{1}^{\prime}$ fundamental wave at $x_{1}=359.89$.

$32 /$ wave $\times 128$ grid points in, respectively, the streamwise and the normal direction. The height of the computational domain is 30 . The grid is similar to that in the previous section, with the stretching parameter $A=0.2$ yielding a ratio $\Delta x_{2 \max } / \Delta x_{2 \min }=34.42$. The maximum aspect ratio of the grid is $\Delta x_{1} / \Delta x_{2}=18.94$. Comparison of different spatial discretization schemes and the influence of a buffer domain on the results is depicted in Figs 8 and 9, respectively, for $u_{1}$ and $u_{2}$ disturbances. In these figures, two groups of lines corresponding to whether a buffer domain is applied (B1) or not (B0) are shown. Calculations without buffer domain exhibit strong standing

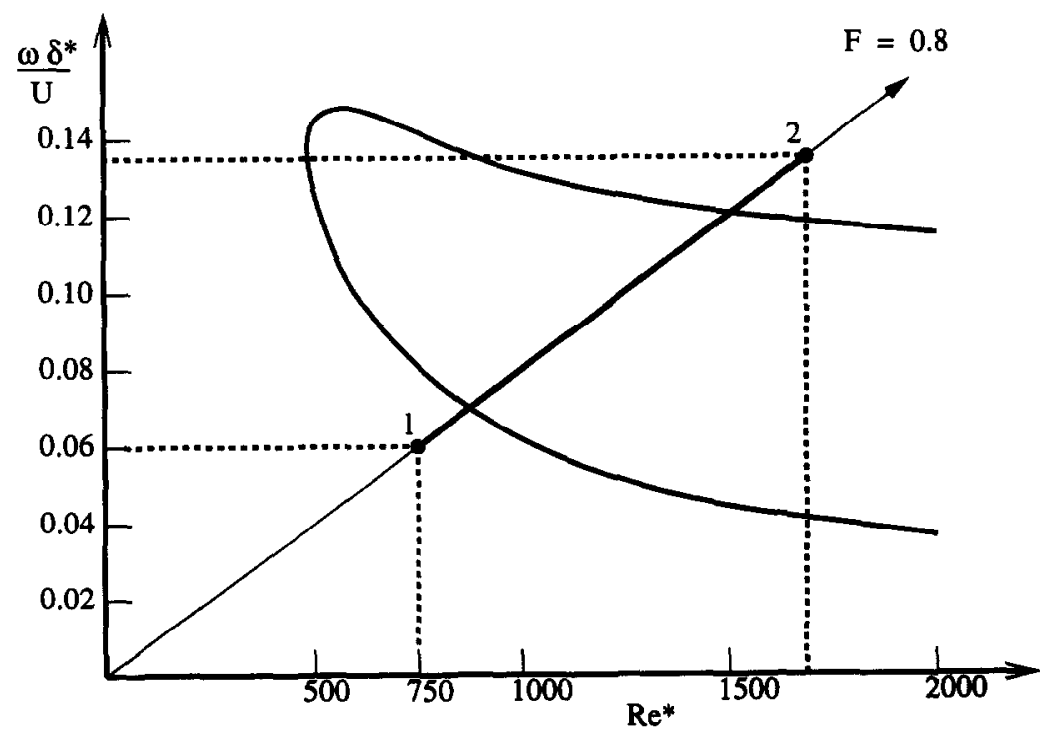

Fig. 13. Position of the computational domain relative to the stability diagram of linear stability theory. 


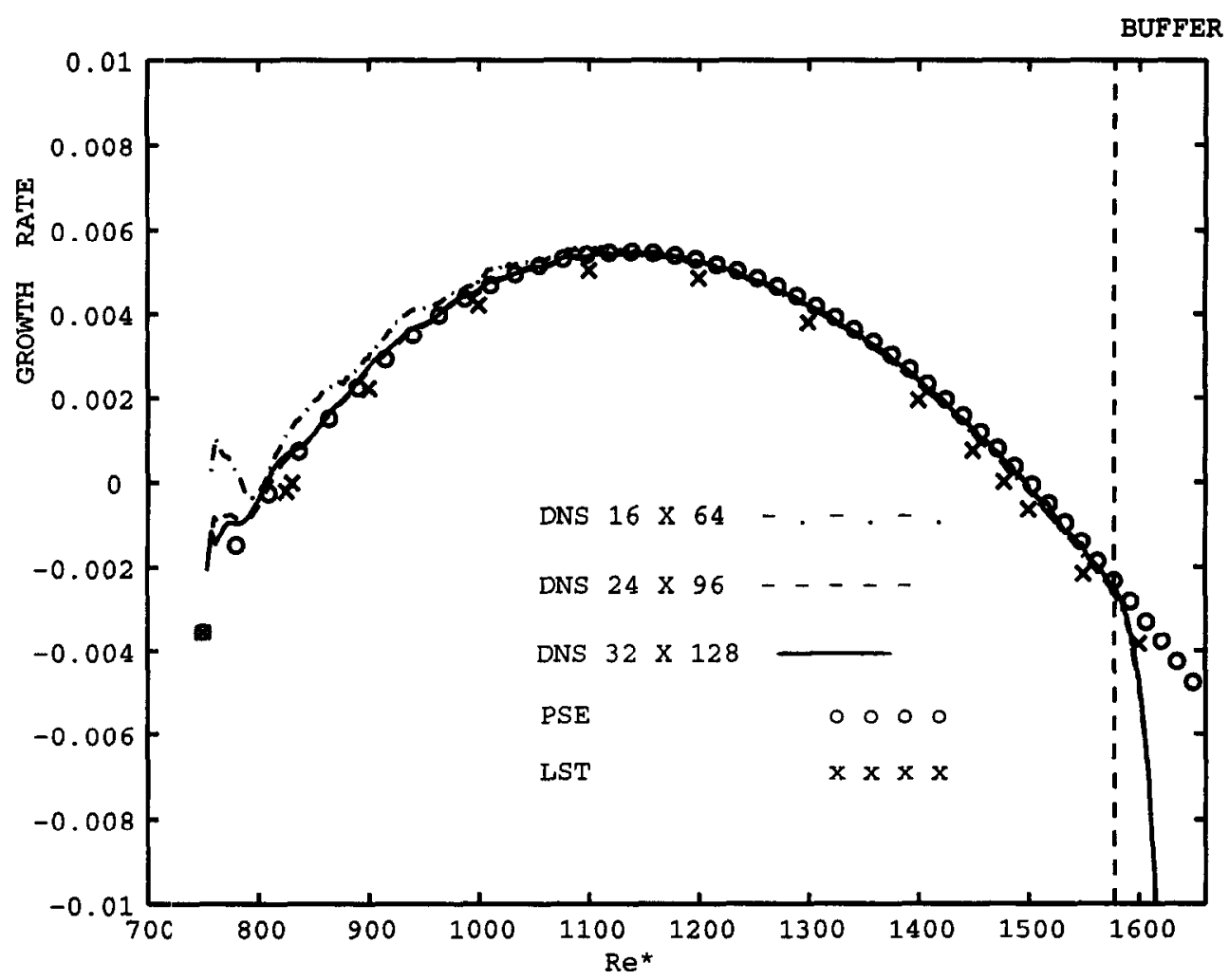

Fig. 14. Comparison of growth rate between DNS, PSE and locally parallel LST. Three DNS results, taken from the $u_{2}^{\prime}$ component, correspond to three different grids.

waves in the amplitude growths of disturbances for both the second order and the fourth order method. Reducing the time steps and increasing the grid density have no appreciable influence on the results, indicating that the observed waves are mainly due to the inflow-outflow boundary coupling and not related to the accuracy of the time integration and the spatial discretization. The indication that the observed waves are standing waves is confirmed by varying the length of the computational domain, which results in a different shape of these waves. Comparison with the corresponding results of a supersonic flow in the previous section shows that an entirely subsonic boundary layer provides more wave reflections than a partly supersonic one. Subsonic flows, contrary to supersonic, have a downstream domain of influence. This implies that some information from outside the flow domain is required. In most applications, such as ours, this information can only be obtained by some approximations. The lack of exact information downstream of the outflow boundary results in wave reflections. To damp these reflections the buffer domain treatment described in Section 4 is applied. The standing waves are substantially suppressed due to the buffer treatment, as illustrated in Figs 8 and 9. The performance of the second order and the fourth order methods is comparable with respect to the growth of the disturbance amplitude, promoting the use of the second order method over the fourth order due to lower computational cost. However, a comparison of the T-S waves from both methods shows that the second order method results in larger phase errors than the fourth order method. The latter agrees excellently with the result from LST. Supported by this result, we use only the fourth order spatial discretization method in the next calculations. Moreover, the use of a buffer domain treatment is obviously essential for accurate results.

The calculations with buffer domain are performed with $C_{1}=0$ and $C_{2}=10$ in equation (24) and $C_{3}=330$ in equation (26). The latter value corresponds to $C_{3} \omega \Delta t \approx 1$. This value of $C_{3}$ is kept the same for all calculations employing the buffer domain, since the results are quite insensitive to variations in $C_{3}$. We proceed with the calibration of $C_{1}$ and $C_{2}$. Much higher or lower values of $C_{2}$ deteriorate the performance of the buffer domain as illustrated by the amplitude growths depicted in Fig. 10. This can be explained as follows. A very low $C_{2}$ suppresses the disturbance amplitude directly after the start of the buffer domain which leads to larger upstream influences, 
whereas a very high $C_{2}$ reduces the amplitude only near the outflow boundary. A large increase of $C_{1}$ has the same effect as a decrease of $C_{2}$, as illustrated by the amplitude growths shown in Fig. 11. A small positive $C_{1}$, for example 0.01 , results in a slight improvement in the growth rate, but as other results do not change, we use $C_{1}=0$ and $C_{2}=10$ in the case of small disturbances. It is, however, expected that the influence of the variation of the damping function parameters on the results will become more appreciable if we consider large disturbances.

The performance of the buffer domain is further validated by varying the length of the buffer domain. Increasing this length to two $\mathrm{T}-\mathrm{S}$ waves yields a slight improvement in suppressing wave reflections. Extending the buffer domain to more than two T-S waves gives no appreciable further improvement. Furthermore, we also varied the length of the physical domain to examine the upstream influence of the buffer domain. Although small standing waves observed in the amplitude growth are slightly changed by this variation, their amplitude is unchanged, which indicates that the upstream influence of the buffer domain is insignificant.

Finally, we compare the disturbance profile of the fundamental wave $u_{1}$ using the fourth order method with the LST result at a specific streamwise location. The streamwise location is selected such that the comparison is made in the neighbourhood of a local wave extremum. Excellent agreement in this comparison is shown in Fig. 12. As in the previous section, similar results are produced by other components. The buffer domain validated in this subsection, which uses $C_{1}=0$ and $C_{2}=10$, will also be employed for the calculations discussed in the next subsections and further specified when we consider large disturbances.

\subsection{Nonparallel base flow instability}

5.3.1. Small perturbations. In this subsection, the results of calculations for nonparallel flat plate flow in the linear regime are presented. The DNS results are compared with the results of PSE. For the comparison of growth rates, results from locally parallel LST are also incorporated. Disregarding the effect of linearization, we should, however, not attempt exact comparisons for the following reasons. In the PSE method, the upstream influence of instability waves is neglected to parabolize the governing equations for the disturbances, whereas LST completely neglects the

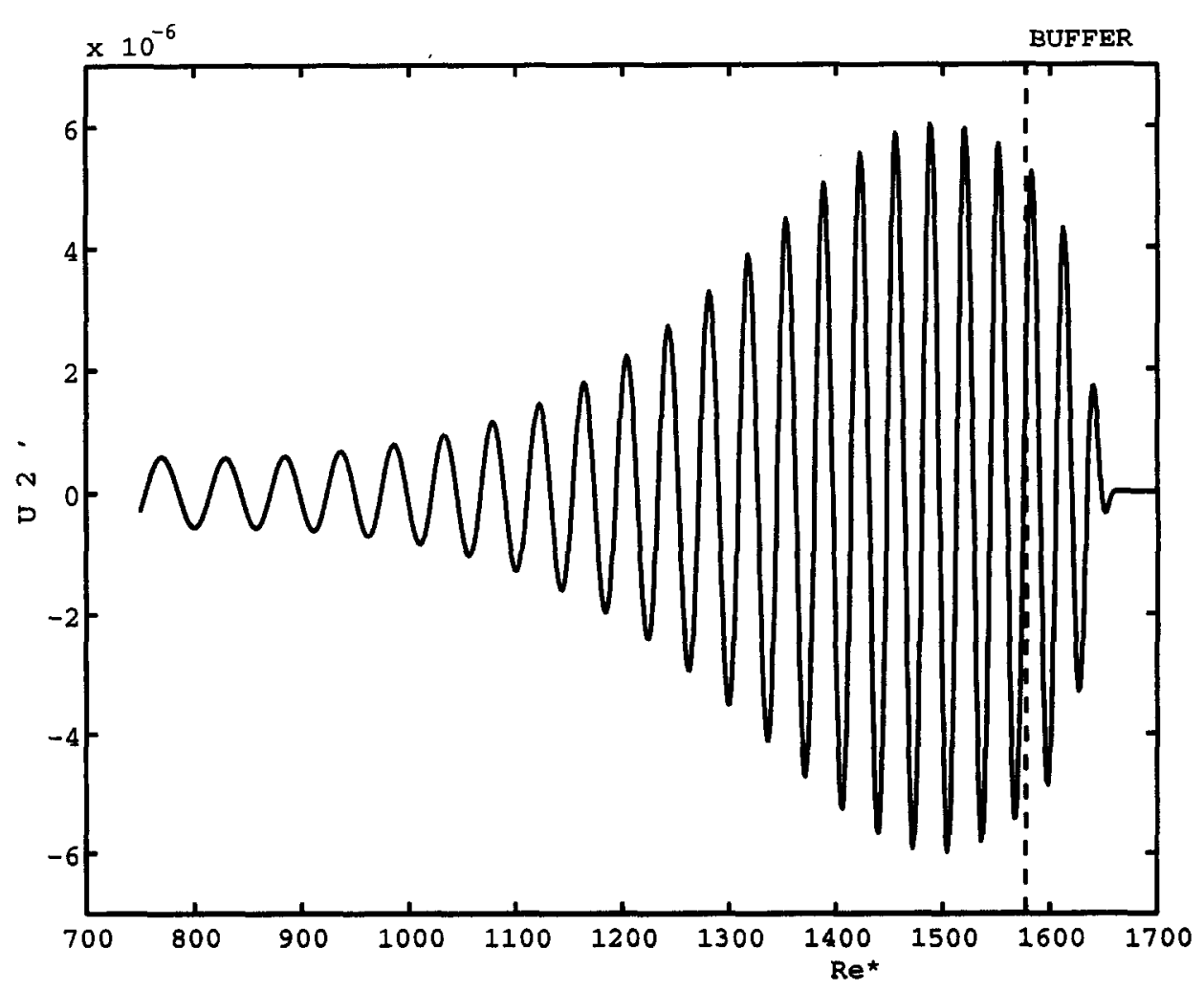

Fig. 15. Development of the $u_{2}^{\prime}$ disturbance in the streamwise direction, taken along $x_{2}=0.1604$. 


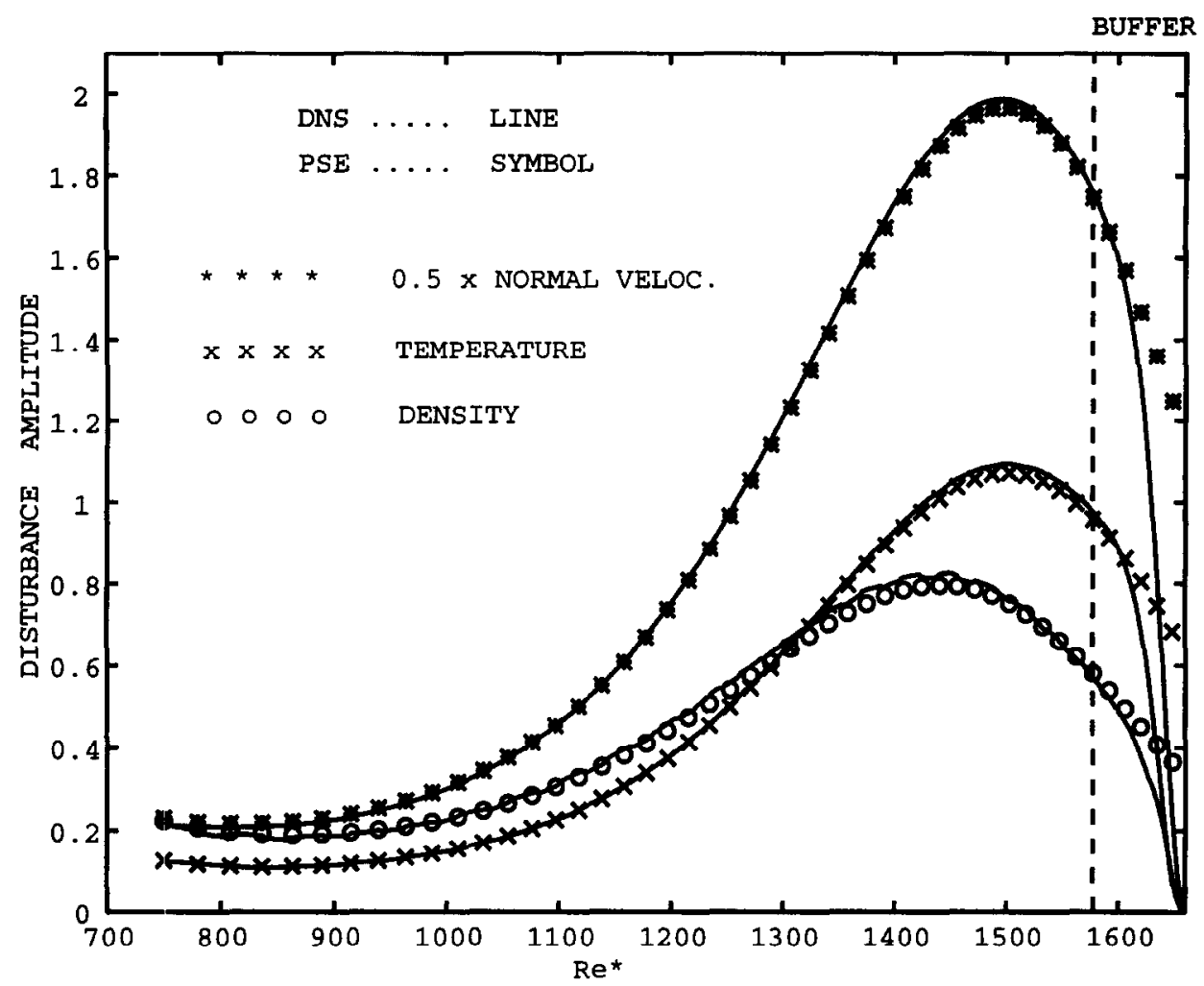

Fig. 16. DNS and PSE disturbance amplitude of various components vs $R e^{*}$.

nonparallel effect caused by the boundary layer growth. In LST, locally parallel flow is assumed using a different length scale, $\delta^{*}\left(x_{1}\right)$, as a consequence of the boundary layer growth. The final results are then rescaled by a reference length.

Specifically, we perform calculations at Mach number 0.5 and reference Reynolds number 750 . The position of the computational domain relative to the stability diagram of linear stability theory is shown in Fig. 13. The test case corresponds to the ray along a dimensionless frequency $F=0.8$, where $F=10^{4} \omega \mu /\left(\rho U_{x}^{2}\right)$. This frequency corresponds to $\omega \delta^{*} / U_{x}=0.06$. The resulting eigenvalue from linear theory is

$$
\alpha=\alpha_{\mathrm{R}}+\mathrm{i} \alpha_{\mathrm{I}}=0.1709+\mathrm{i} 0.003549
$$

corresponding to point 1 in Fig. 13. This is a slightly stable mode close to the lower branch of the neutral curve. As we proceed downstream, the disturbance modes become unstable. The flow domain extends to a local Reynolds number about 2.25 times as large as the inflow Reynolds number, corresponding to a damping mode, somewhat beyond the upper branch of the neutral curve (point 2). The extent of the domain is equivalent to 25 disturbance wavelengths downstream of the inflow boundary. The last four wavelengths define the buffer domain. In the PSE calculations, 100 grid points were used in the wall normal direction, which extends to a height of 80 , and four marching steps were taken per disturbance wavelength in the streamwise direction. Identical disturbances to the inflow of DNS are used to start the PSE calculations.

In contrast with calculations in the parallel flow case, the base flow of the nonparallel flow case is not the same as the similarity solution to the compressible boundary layer, which is used as the initial condition. Two ways can be followed for the specification of the base flow: by performing a steady state calculation first without imposing perturbations and by time averaging in the statistically stationary state. Although it implies that more work should be carried out, we preferred the latter method, for the following reasons. Firstly, performing a steady state calculation first for the base flow is somewhat unpractical and time consuming. Secondly, this method is not generally applicable. For instance for higher Reynolds number cases or flows subjected to adverse pressure gradients, which contain highly unstable modes, it is impossible to obtain a steady state solution using the present method. Small numerical errors will act as noise, which then amplifies as 
propagating physical waves [21]. The third reason is that we want to examine the adequacy of the time averaging procedure, since it can be applied directly to more complex flows, such as separated flows [21] and turbulent flows. The time interval for the averaging process is taken here as one disturbance period, and we repeat this process until the mean field has converged, which takes approximately 10 disturbance periods. The data sampling of the perturbations is subsequently carried out.

To appreciate the effect of the grid density, three different resolutions are used, namely $16 /$ wave $\times 64,24 /$ wave $\times 96$ and $32 /$ wave $\times 128$ grid points. As an illustration of the computational time, the coarsest grid, for instance, requires about $1 \mathrm{~h}$ CPU-time on a single processor of a Silicon Graphics R8000. Growth rates resulting from these grids are compared with results from PSE and locally parallel LST in Fig. 14. The growth rates are taken from the $u_{2}^{\prime}$ component. The Reynolds number $R e^{*}$ against which the growth rates are plotted is based on the local displacement thickness growing in the streamwise direction. Results from other components are, however, slightly more sensitive to the upstream influence of the outflow boundary and exhibit small standing waves around the curve of growth rates taken from $u_{2}^{\prime}$. Small transients occur near the inflow boundary, in which the prescribed inflow adjusts to the solution of the governing system. The results of the grid 16/wave $\times 64$ exhibit rather large deviations from the results of the finest grid, whereas using the grid 24/wave $\times 96$ leads to nearly equivalent results. The result of PSE is closer to DNS than the result of locally parallel LST. The growth rates from LST are systematically lower than those of PSE. The same phenomenon has also been observed by Bertolotti in Ref. [22] and Fasel in Ref. [24]. Noting that the growth rate is a very critical comparison parameter, the agreement between DNS and PSE is very well in spite of the fact that the comparison is performed within a subsonic regime which is more critical for inflow and outflow boundary conditions. In the literature, comparison between DNS and PSE is commonly carried out in supersonic flows such as those presented in Refs [3,11]. A similar comparison between DNS and PSE at a subsonic Mach number was carried out by Van der Vegt in Ref. [10]. The current results compare favourably with the latter, especially regarding the upstream influence of the buffer domain which is very small in the present results. The maximum relative difference in the phase of $\mathrm{T}-\mathrm{S}$ waves resulting from grid $24 /$ wave $\times 96$ and grid 32 /wave $\times 128$ is also small, about $0.6 \%$. The relative difference of the phase

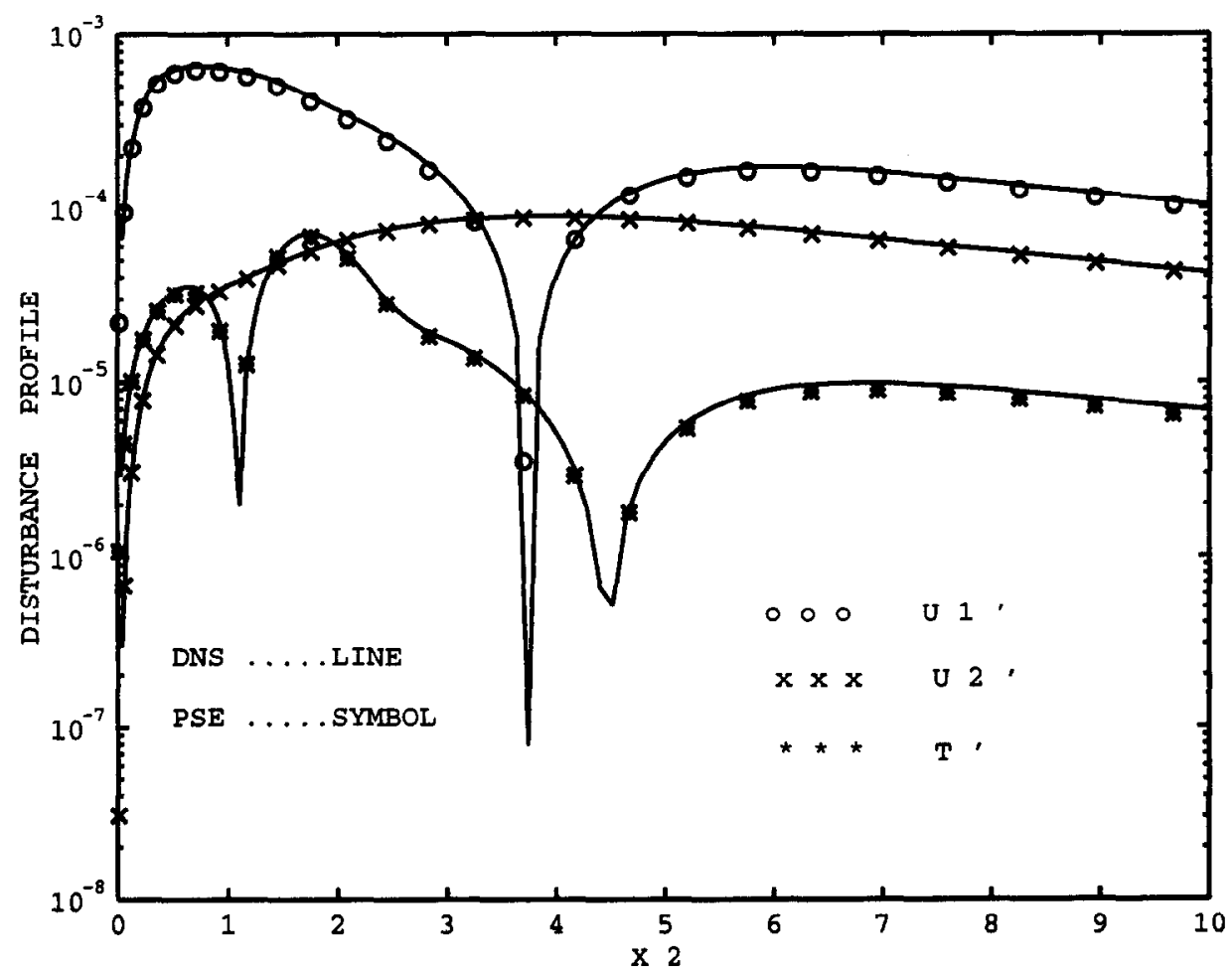

Fig. 17. PSE and DNS disturbance profiles at $x_{1}=988.34(R e=1570.2)$. 

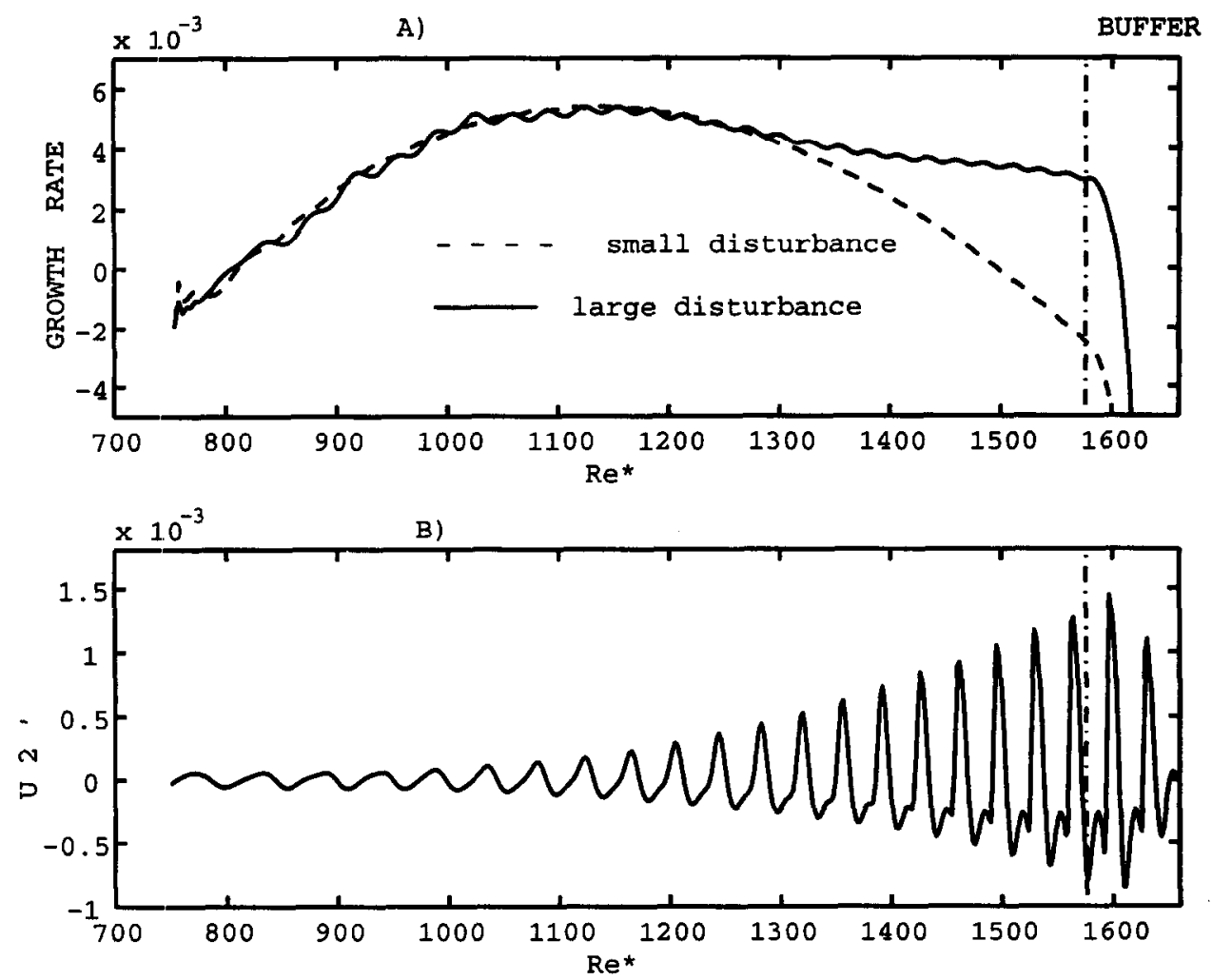

Fig. 18. (A) Comparison of growth rates, taken from the $u_{2}^{\prime}$ component, resulting from small and large disturbances. (B) Development of the $u_{2}^{\prime}$ disturbance along $x_{2}=0.1604$ in the streamwise direction.

is defined as the shifting of the $\mathrm{T}-\mathrm{S}$ waves in the streamwise direction relative to one wavelength. It appears sufficient to use the coarser grid for the nonparallel test case. This phase difference is not appreciable if we plot the $\mathrm{T} S$ waves of the two finer grids in the same figure, therefore only the T-S waves of the grid $24 /$ wave $\times 96$ are presented in Fig. 15, showing the development of the $u_{2}^{\prime}$ disturbance in the streamwise direction. Corresponding to the development of the growth rates, which shows successively negative, positive and again negative $\alpha_{i}$ values as a function of $x_{1}$, the disturbance decays, grows up to its maximum amplitude, and then decays again rapidly to zero. The decay near the outflow boundary is partly physical and within the buffer domain it is further increased by the buffer treatment. Note that in spite of the physical damping, the disturbance amplitude is still quite large when entering the buffer domain.

In contrast to the growth rates $\alpha_{i}$ which are the same for all components, the development of the disturbance amplitude in the $x_{1}$ direction is different per component. This is clarified by considering the disturbances analogous to equation (8) for nonparallel flows:

$$
v=\epsilon \mathrm{e}^{-\int x_{i} \mathrm{dx}}\left(\psi_{\mathrm{R}}^{r} \cos \left(\int \alpha_{\mathrm{R}} \mathrm{d} x_{1}-\omega t\right)-\psi_{\mathrm{I}}^{r} \sin \left(\int \alpha_{\mathrm{R}} \mathrm{d} x_{1}-\omega t\right)\right) .
$$

The corresponding amplitude of a disturbance component $v_{i}$ as a function of $x_{1}$ is now

$$
R_{i}=\epsilon \mathrm{e}^{-\int \alpha_{i} \mathrm{~d} x_{1}} \sqrt{\left(\psi_{\mathrm{R}}^{i}\right)^{2}+\left(\psi_{\mathrm{i}}^{\mathrm{i}}\right)^{2}}
$$

Here, besides the growth rates $\alpha_{i}$, the functions $\psi_{\mathrm{R}}$ and $\psi_{1}$ also depend on $x_{1}$. Moreover, this dependence is unique per component. Hence in a nonparallel flow we expect that the streamwise position of the maximum amplitude for various components is not necessarily identical. For this reason it is important, along with growth rate comparison, to compare also the streamwise development of the disturbance amplitude of DNS and PSE corresponding to different 
components. This comparison is shown in Fig. 16. As expected, different disturbance components exhibit different streamwise developments of amplitude and different streamwise positions of global maxima. Although the maximum amplitudes of all components resulting from the PSE slightly underestimate those of the DNS, the DNS results generally compare well with the PSE results.

Similar to the previous sections, we also compare the disturbance profiles of the DNS with the corresponding PSE results. This comparison is shown in Fig. 17, presented as the absolute value of disturbance components $u_{1}^{\prime}, u_{2}^{\prime}$ and $T^{\prime}$. We perform the comparison at a streamwise location corresponding to $R e^{*}=1570.2$ which is beyond the amplitude maxima and near the beginning of the buffer domain. The complex structure of the disturbance profiles is accurately captured, and a critical examination shows that the PSE results again slightly underestimate the DNS results. Performing the comparison at various streamwise locations yields similar results, indicating that the upstream influence of the buffer domain is insignificant and that the observed deviation is not related to the buffer domain. In contrast with parallel flow comparisons between DNS and LST, in which we are certain that the modelling error due to linearization is in the order of the square of the disturbances, here we face an uncertainty regarding the physical effects of the approximation beyond linearization in the PSE method, namely the parabolization. Although the parabolizing procedure is applied only to the shape functions (disturbance profiles), whereas the formulation for the wave parts, $\alpha_{\mathrm{R}}$ and $\alpha_{i}$, remain elliptic, this might form an explanation for the slight underestimation of the PSE. As a consequence of neglecting upstream influences in the PSE method, we might expect a better agreement between DNS and PSE when we consider higher Mach numbers, since the upstream influences are smaller with increasing Mach numbers.

5.3.2. Large perturbations. A nonparallel flow simulation in the nonlinear regime is conducted by exactly repeating the linear case with an increased disturbance amplitude $\epsilon=0.01$. The grid 32 /wave $\times 96$ is used to capture the higher amplitude of the fluctuations. The effects of nonlinearity are examined by comparing the results with those of the linear case. A comparison of growth rates between the linear and nonlinear case is shown in Fig. 18(A), taken from the $u_{2}^{\prime}$ component. In the beginning, the growth rates of the large disturbances roughly follow those of the small disturbances, but after reaching a maximum value, contrary to the linear case, they exhibit only
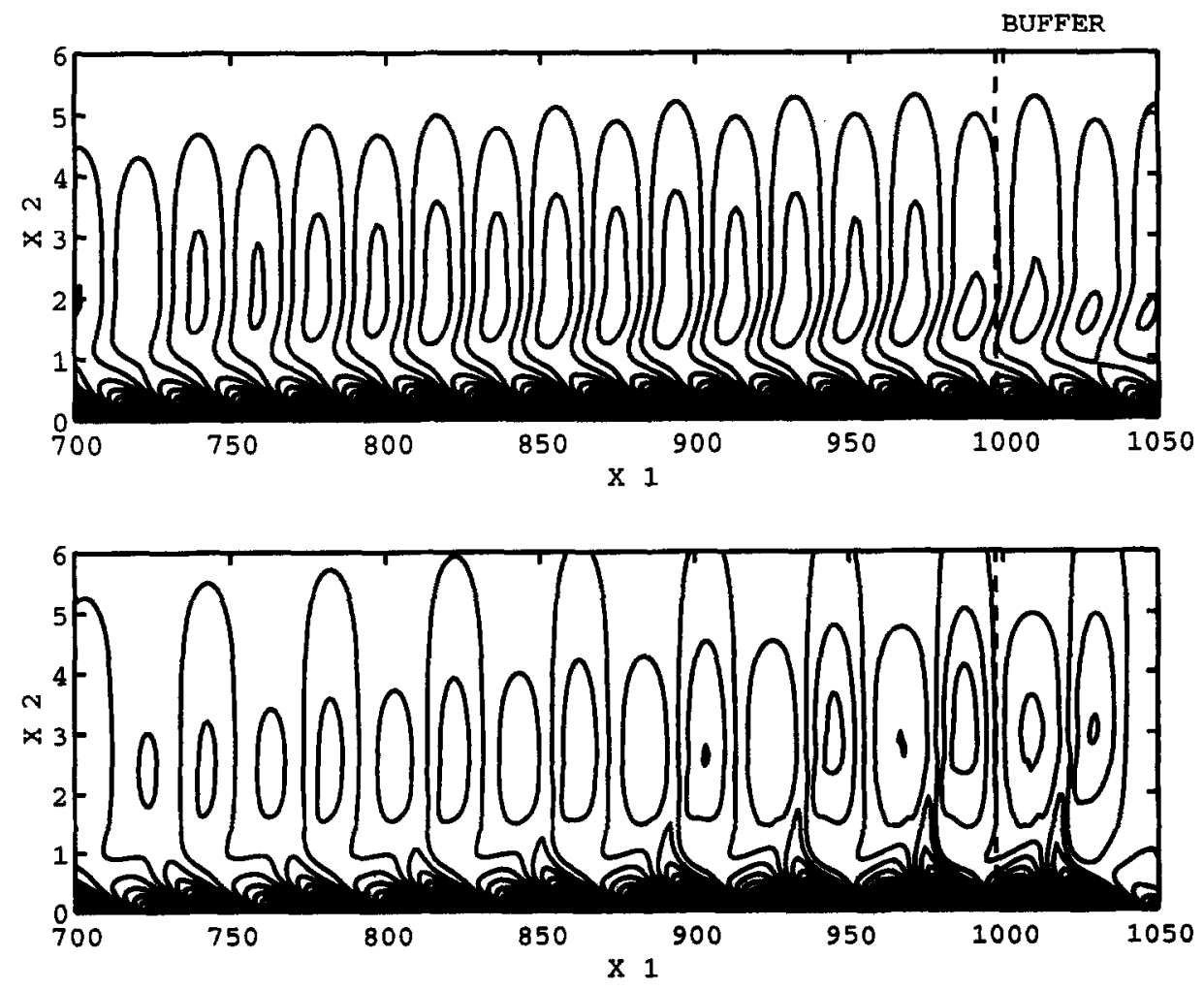

Fig. 19. Disturbance vorticity contours for the linear case (above) and nonlinear case (below). 


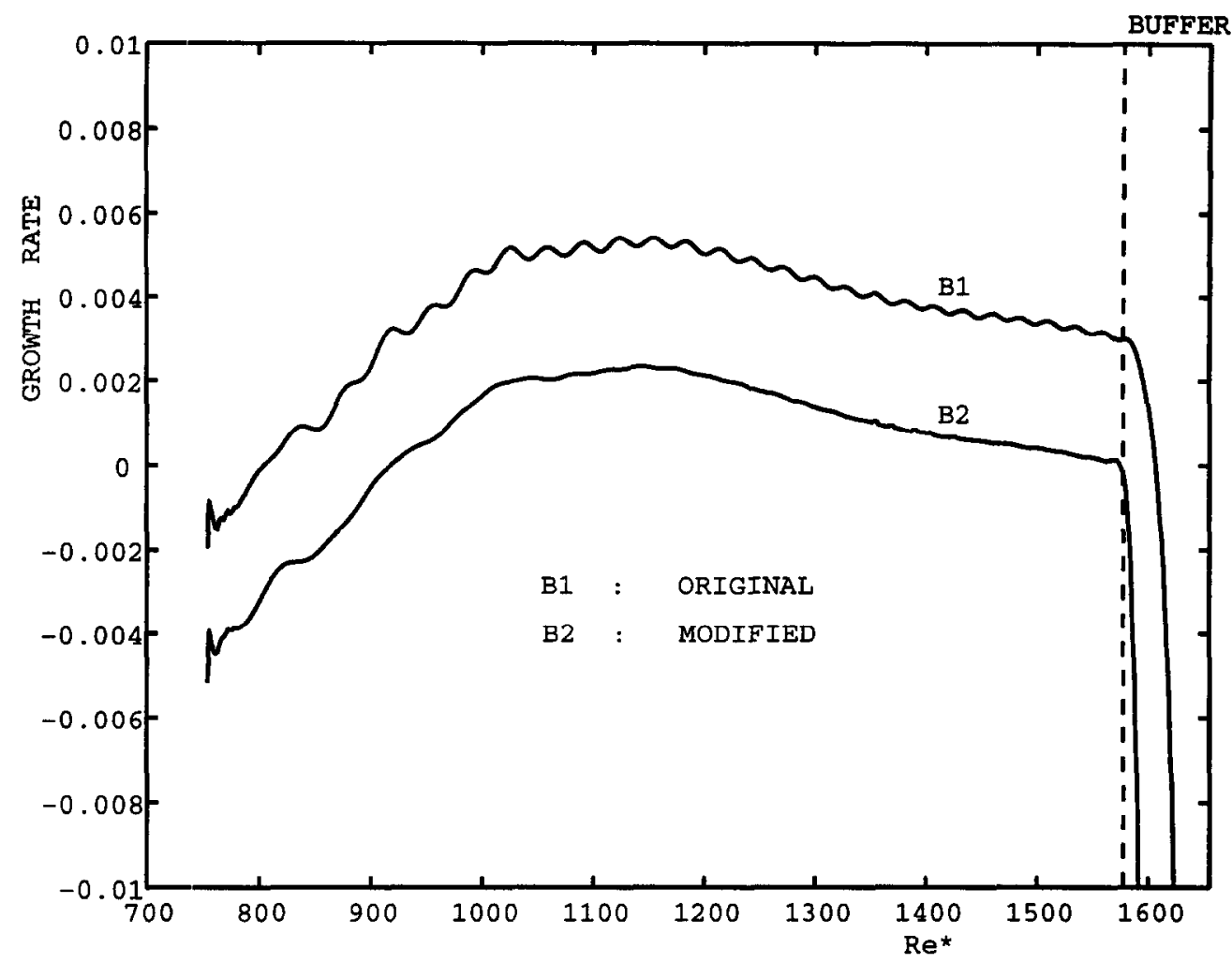

Fig. 20. Comparison of growth rates, taken from the $u_{2}^{\prime}$ component, in the nonlinear case resulting from the original parameter setting (B1) and the new setting (B2).

a small decrease and end up still growing considerably $\left(\alpha_{i}<0\right)$ before entering the buffer domain. The deviation from the linear case is also enhanced by the occurrence of small standing waves in the growth rates of the nonlinear case, indicating that flow with large disturbances experiences larger upstream effects in spite of the buffer domain treatment. A nonlinear effect is also appreciable in the shape of the T-S waves. This is illustrated in Fig. 18(B) by plotting the same T-S wave as in Fig. 15. The nonlinear $\mathrm{T}-\mathrm{S}$ wave exhibits an irregular pattern in the streamwise direction instead of a sinusoidal form as in the linear case. Besides, it grows monotonically up to the buffer domain. A nonlinear effect can be seen as well if we compare the mean flow of the linear and nonlinear cases. We can see a deformation in the perturbation vorticity contours due to the nonlinearity in Fig. 19. The absolute value of the perturbation vorticity for the linear case decreases already upstream of the buffer domain associated with damping modes, whereas the corresponding value in the nonlinear case grows monotonically until the buffer domain. Considering the sign of the perturbation vorticity, it consists of positive and negative parts, marching alternately in the streamwise direction. Contrary to the linear case, in which the positive and negative parts of the perturbation vorticity are nearly equal, the negative part in the nonlinear case is more pronounced than the positive part.

Regarding the occurrence of small standing waves in the growth rates of the nonlinear case, a further investigation has shown that this is due to a much larger decrease of the disturbance amplitude within the buffer domain than in the linear case. The larger amplitude must be suppressed to zero within the same buffer domain length. Consequently, we should employ a higher grid density in the buffer domain or extend the length of the buffer domain to anticipate this amplitude reduction. However, this implies a higher computational cost. A more elegant remedy, avoiding an increase in the computational cost, is to attempt a more gradual decrease of the damping function. This can be achieved by activating the parameter $C_{1}$ in the damping function, equation (24), forcing a more gradual suppression of disturbances in the front part of the buffer domain, and at the same time increasing the value of $C_{2}$, postponing a rapid decrease to zero in the rear part. $C_{1}=0.005$ and $C_{2}=20$ are found to be appropriate values. The performance of the damping function due to this alternative tuning is demonstrated by comparing the growth rates 
of the nonlinear case resulting from the two sets of parameter in Fig. 20. This comparison illustrates a substantial improvement due to a more gradual decrease of the damping function. Note that the growth rates resulting from $C_{1}=0.005$ and $C_{2}=20$ directly after entering the buffer domain decrease more rapidly due to a lower value of the damping function in the interior of the buffer domain. Testing the damping function using these new values of damping function parameters in the case of small disturbances we found a slight improvement in the growth rates but inappreciable effects in other comparison parameters. Based on this result, the new parameter values can be used in both linear and nonlinear regimes. Finally, we notice that the effects of nonlinearity discussed above become more profound when increasing the amplitude of forced disturbances.

\section{CONCLUSION}

In this paper, numerical methods were developed that are suitable for direct numerical simulations of compressible laminar-turbulent transition in boundary layers. The important aspects of these methods are the fourth order central difference approximation and the specification of outflow boundary treatments. The fourth order scheme yields considerably more accurate results than the second order scheme. In flow simulations with time dependent perturbations, spurious reflections are generated by the inflow and outflow boundary conditions. The coupling between these boundaries results in the formation of standing waves in the interior domain. This distortion becomes large with increasing amplitude of disturbances and can even result in a divergent solution. The wave reflections can, however, be significantly damped by applying a buffer domain treatment in addition to the outflow boundary condition. On the other hand, the upstream influences become smaller with increasing Mach numbers. Hence, this buffer domain treatment is not required if we consider high supersonic Mach numbers.

This buffer approach employs a direct reduction of the fluctuations of the solution components within the buffer domain, using a damping function. A previous investigation confirms that this direct approach is more efficient than other, indirect approaches such as increasing viscosity, parabolizing procedure, base flow acceleration and increasing grid spacing [8]. The validation of the damping function is performed by testing the buffer domain in successively more difficult test cases. In this way the domain of the damping function parameters is narrowed and the eventually selected values of these parameters are applicable for both the linear and nonlinear regimes. Numerical experiments show that the present buffer domain approach exhibits very small upstream influences. Moreover, it is insensitive to the extent of the domain of interest, the reference parameters and the grid density.

The calculation results of flows with small disturbances using the above numerical method compare very well with the results of LST and PSE. In agreement with the observations of other authors, the locally parallel assumption of LST underestimates the growth of disturbances in a nonparallel flow. The good agreement between results of the DNS and the PSE supports the use of shape functions resulting from PSE as the imposed disturbances of DNS. The numerical methods have also been successful in performing calculations with large disturbances, proceeding the flow into the nonlinear regime. Although, contrary to the outflow boundary, no additional treatment is applied to the inflow boundary conditions to damp spurious reflections generated by this boundary, the error near the inflow boundary is small. This satisfactory result encourages the use of the employed numerical methods in more complex flows such as flows which proceed into the turbulent regime and flows with separation regions [21]. These applications form the subject of future work within the framework of 3D DNS and large eddy simulation of compressible turbulent flow.

Acknowledgements-This work was financially supported by the Koninkelijke Nederlandse Academie van Wetenschappen (Royal Netherlands Academy of Sciences, KNAW). The authors would also like to thank R. S. Heeg for the PSE code provided.

\section{REFERENCES}

1. Laurien, E. and Kleiser, L., Numerical simulation of boundary-layer transition and transition control. Journal of Fluid Mechanics, 1989, 199, 403-440.

2. Zang, T. A. and Hussaini, M. Y., Numerical simulation of nonlinear interactions in channel and boundary layer transition. In Nonlinear Wave Interactions in Fluids, eds R. W. Miksad, T. R. Akylas and T. Herbert. AMD 87, ASME, New York, 1987, pp. 131-145. 
3. Guo, Y., Kleiser, L. and Adams, N. A., A comparison study of an improved temporal DNS and spatial DNS of compressible boundary layer transition. AIA A Journal, 1994, 94, 2371.

4. Rudy, D. H. and Strikwerda, J. C., Boundary conditions for subsonic compressible Navier-Stokes calculations. Computers and Fluids, 1981, 9, 327-338.

5. Thompson, K. 'W., Time dependent boundary conditions for hyperbolic systems. Journal of Computational Physics, $1987,68,1-24$.

6. Poinsot, T. J. and Lele, S. K., Boundary conditions for direct simulations of compressible viscous flows. Journal of Computational Physics, 1992, 101, 104-129.

7. Strikwerda, J. C., Initial boundary value problems for incompletely parabolic systems. Communications in Pure and Applied Mathematics, 1977, 33, 797-822.

8. Wasistho, B., Creurts, B. J. and Kuerten, J. G. M., Artificial boundary treatments for the simulation of viscous compressible flow over a flat plate. Proceedings of 7th IASE Conference, UMIST, 1995.

9. Liu, Z. and Liu, C., Fourth order finite difference and multigrid methods for modeling instabilities in flat plate boundary layers - 2D and 3D approaches. Computers and Fluids, 1994, 23, 955-982.

10. Van der Vegt, J. J. W., Higher order accurate Osher schemes with application to compressible boundary layer stability. AIAA Paper, 93-3051, 1993.

11. Pruett, C. D., Zang, T. A., Chang, C. L. and Carpenter, M. H., Spatial direct numerical simulation of high-speed boundary-layer flows, part I, algorithmic consideration and validation. Theoretical Computational Fluid Dynamics, $1995,7,49-76$.

12. Streett, C. L. and Macaraeg, M. G., Spectral multi-domain for large-scale fluid dynamic simulations. Applied Numerical Mathematics, 1989-1990, 6, 123-139.

13. Kloker, M. and Konzelmann, U., Outflow boundary conditions for spatial Navier-Stokes simulations of transition boundary layers. AIAA Journal, 1993, 31, 620-628.

14. Stewartson, K., The Theory of Laminar Boundary Layers in Compressible Fluids. Oxford Mathematical Monographs, Oxford, 1964.

15. Vreman, A. W., Geurts, B. J. and Kuerten, J. G. M., Comparison of numerical schemes in large eddy simulation of the temporal mixing layer. International Journal for Numerical Methods in Fluids, 1996, 22, 297-311.

16. Vreman, A. W., Geurts, B. J. and Kuerten, J. G. M., Large eddy simulation of the turbulent mixing layer. Journal of Fluid Mechanics, in press.

17. Vreman, A. W., Geurts, B. J., Kuerten, J. G. M., Broeze, J. and Wasistho, B., Dynamic subgridscale models for LES of transitional and turbulent compressible flow in 3-D shear layers. Proceedings of Tenth Symposium on Turbulent Shear Flows, Pennsylvania, 1995.

18. Gresho, P., Inccmpressible fluid dynamics, some fundamental formulation issues. Annual Review of Fluid Mechanics, $1991,23,343-370$.

19. Blaisdell, G. A., Mansour, N. N. and Reynolds, W. C., Compressibility effects on the growth and structure of homogeneous turbulent shear flow. Journal of Fluid Mechanics, 1993, 256, 443-485.

20. Jameson, A., Transonic flow calculations. MAE-Report, 1651, Princeton University, 1983.

21. Wasistho, B., Geurts, B. J. and Kuerten, J. G. M., Numerical simulation of separated boundary-layer flow. Journal of Engineering Mathematics, in press.

22. Bertolotti, F. P., Linear and nonlinear stability of boundary layers with streamwise varying properties. Dissertation, Ohio State University, Columbus, OH, 1990.

23. Mack, L. M., Boundary layer stability theory. In Special Course on Stability and Transition of Laminar Flow, AGARD Report 709, 1984.

24. Fasel, H., Investigation of the stability of boundary layers by a finite-difference model of the Navier-Stokes equations. Journal of Fluid Mechanics, 1976, 78, 355-383. 